\title{
RISK CONTROL MECHANISMS OF THIRD-PARTY GUARANTEE WHEN FINANCING NEWSVENDOR
}

\author{
Jiazhou Huang ${ }^{1}$, Qihui Lu ${ }^{2,3, *}$, Xiangfeng Chen ${ }^{1}$ And Jizhou Zhan ${ }^{4}$
}

\begin{abstract}
Two risk control mechanisms, namely, cash deposit, and loan limits based on retained profit or maximum expected guarantor profit, are developed for capital-constrained newsvendor financing. Results show that with a large initial capital or mortgage asset, the newsvendor reduces the order quantity to avoid bankruptcy risk. Under the risk control mechanism with a cash deposit, the guarantor gains greater profit when the newsvendor has a low initial capital. Setting a loan limit is thus an effective mechanism for newsvendors to not only reduce the bankruptcy risk, but also increase the guarantee profit. Numerical experiments also show that, compared with the risk control mechanism based on maximum expected profit, loan limits based on retained profit is more beneficial for the newsvendor, guarantee, and the bank.
\end{abstract}

Mathematics Subject Classification. 90B06.

Received March 11, 2021. Accepted July 14, 2021.

\section{INTRODUCTION}

Small and medium-sized enterprises (SMEs) play a significant role in the economic growth and job creation in both developed and developing countries. In 2018, 30.2 million SMEs in the United States (US) occupied $99.9 \%$ of businesses, created 1.9 million net new jobs, and employed 58.9 million workers, which is nearly half of the total country workforce [27]. However, many SMEs face capital constraints, and thus require short-term financing to continue running the business because of high operating cost. Supply chain disruption occurs when SMEs have no sufficient capital for operations. For example, a capital-constrained manufacturer restricts its own order quantity and fails to meet retailer's order demands. Meanwhile, SMEs face difficulties to obtain financial resources in capital markets because of their low quality or poor history credit, for example, only $20 \%$ of all SMEs can obtain loans from commercial banks in China [23]. Moreover, by the estimation from the US, a decreasing number of commercial banks are willing to provide loans for SMEs [10].

Simultaneously, banks are also interested in selecting safe borrowers, thus engaging in secure and profitable transactions. Therefore, to meet the financing needs of SMEs that lack access to financial institutions, a common

Keywords. Supply chain finance, risk control, newsvendor financing, guarantee.

1 School of Management, Fudan University, Shanghai 200433, P.R. China.

2 School of Business Administration, Zhejiang Gongshang University, Hangzhou 310018, P.R. China.

3 Research Center of Modern Business and Trade, Zhejiang Gongshang University, Hangzhou 310015, P.R. China.

4 School of Business, Nanjing Audit University, Nanjing 211815, P.R. China.

*Corresponding author: qihuilu@163.com

(c) The authors. Published by EDP Sciences, ROADEF, SMAI 2021 
solution for credit enhancement is loan guarantee finance, which is becoming more prevalent in financially distressed supply chains. With the innovation and evolution of financial business modes, Evergrowing Bank, Hua Xia Bank, and Industrial and Commercial Bank of China carried out an innovative scheme. These banks lend funds to capital-constrained retailers based on the credit guarantee of upstream manufacturers [12, 16, 28]. Third-party guarantees are one form of securing loans, where the guarantor is liable for the outstanding debt including interest, in case the borrower defaults. Guarantors should repay the remaining balance when the original borrowers cannot repay the loan and the respective interest in full. Thus, loans become more attractive to potential lenders. Well-structured financing schemes have proven to be valuable and indispensable for accelerating the turnover of inventories and receivables to provide more loans. More appropriately, obtaining guarantees from the third-party while engaging in supply chain finance (SCF), banks can now provide more loans with lower interest rate for SMEs, which reduce the risk of bad loans. SMEs often face a problem similar to that of a newsvendor because of uncertain market demand, and thus in this study, SMEs are considered as newsvendors.

This financing scheme is widely used in agriculture. In recent years, the agriculture sector has received prominent importance in many developing countries. Agriculture also traditionally employs a large labor force in rural areas where poverty has been a long-standing social problem. The consumption and trade of agriculture products have increased as rapidly as economic and population growth. However, farmers in these regions remain poor due to the lack of opportunities to improve their operations. The primary reason is that most farmers are smallholders with little initial capital for production. In the US, the debt level of farmers causes so much concern, especially for younger and smaller farms that their viability comes into question. In the European Union, farmers exhibit lower levels of debt at declining interest rates, and are less likely to face financial distress in the near future. Given the lack of money, farmers have no access to purchase sufficient raw materials for production. The other severe problem is that most capital-constrained farmers cannot obtain sufficient loans from traditional business banks for production. For these farmers, credit information systems are too imprecise in collecting past credit information. Moreover, the fluctuating yield of agricultural products also brings high default probability. If farmers go bankrupt, repaying the bad loan becomes very difficult. All of these issues result in the difficulty of banks to implement risk assessments on farmers.

Third-party guarantees can mitigate the gap between the farmers and commercial banks. In the agricultural supply chain, we take the "Hope Financing", which is initiated by the New Hope Group in China as an example. The New Hope Group is an industrialized agricultural and leading enterprise in China and Hope Financing is a financing platform connecting the capital-constrained farmers and lenders. Clearly, lenders bear the huge risk of bad loan when the farmer becomes bankrupt. This risk reduces the willingness of lenders to provide farmers with loans, which in turn, can damage the profit of leading enterprises of the total supply chain. To overcome this difficulty, the Chinese government set an alliance called "State Agricultural Credit Guarantee Alliance" as the third-party to guarantee the farmer loans in 2016. This alliance established a complete system of guarantee and re-guarantee organizations dedicated to supporting the moderate agricultural operations, sharing the loan risks of banks and reducing the comprehensive credit costs actually borne by farmers. In case farmers become bankrupt, lenders can obtain the entire revenue of farmers and then the loss are repaid by the third-party guarantees.

The third-party guarantee provides assurance for many newsvendors. Although several newsvendors become bankrupt, the total guarantee fee can cover and repay the bad loans. Therefore, the third-party guarantee still has an incentive to undertake loans of newsvendors with certain risk of bankruptcy. However, the rate of bad loans is annually increasing in China, from $2.17 \%$ in 2015 to $2.78 \%$ in $2017^{1}$. Moreover, the number of third-party guarantees in the market is decreasing, from approximately 8590 in 2012 to 8025 in 2017 and 5937 in $2019^{2}$. Thus, providing financing guarantees for newsvendors presents huge risks.

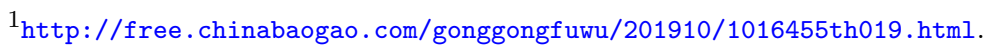

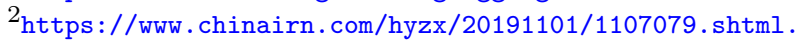


The above observations suggest the importance of third-party guarantees in helping a capital-constrained newsvendor to mitigate the financing gap and improve their profits. In addition, taking an effective risk control mechanism is critical for the development of the third-party guarantee. Despite the popularity of such schemes, their impact on the profit of SMEs or the third-party guarantee remains unclear. Consequently, this study examines the risk control mechanisms of third-party guarantees for capital-constrained newsvendors. The research questions are as follows.

(1) How do the initial capital and market demand affect the optimal solution of capital-constrained newsvendors and the preference of risk control mechanism of the third-party guarantee?

(2) Can both the newsvendor and the third-party guarantee obtain more profits with the loan limit mechanism?

To answer the above questions and to better understand the impact of third-party guarantee on newsvendors, we investigate an innovative financing mode motivated by real businesses. A two-stage model is developed as benchmark, in which a capital-constrained newsvendor decides on order quantity at the beginning of selling season. If the production cost is beyond the initial capital, the newsvendor can borrow funds from a commercial bank. At the end of sales season, the newsvendor collects all revenue from the realized market demand and then pays off the loan and interest to the commercial bank. In case the total revenue of newsvendor cannot cover the repayment, the third-party guarantee covers for the bad loan. In this study, a guarantee financing model is established with a capital-constrained newsvendor, commercial bank, and a third-party guarantee. Two main risk control mechanisms are designed, namely, with cash deposit and with loan limit. Two methods of setting loan limit are also designed on the basis of a retained or a maximized profit. Then, the optimal order quantity of newsvendors and the expected profit of the third-party guarantee are explored under different risk control mechanisms. Interesting results are obtained from the combined analytical solutions and numerical study.

The remainder of the paper is organized as follows. The next section provides a brief survey on related literature. Section 3 describes the basic financing model in the supply chain with the third-party guarantee. Sections 4 and 5 present the risk control mechanisms of cash deposit and loan limit, respectively. A numerical study is carried out to analyze the impact of the two risk control mechanisms on the rate of bad loan of a commercial bank and the total profit of the third-party guarantee in Section 6. Conclusion and future research are provided in Section 7. All Proofs are presented in Appendix A.

\section{Literature REVIEW}

This study is related to multiple research streams in the growing supply chain finance literature. The first research stream relates and contributes to literature that explores the intersection of finance and decisions in operations management. Buzacott and Zhang [5] first discussed the operational and financial decisions of a capital-constrained retailer and found that compared with using their own capital, using asset-based financing enables retailers to enhance their returns. Kouvelis and Zhao [14] checked the optimal price-only contract when selling to a cash-constrained newsvendor when bankruptcy is costly. Deng et al. [9] showed that supplier initial capitals, production costs, and heterogeneities can influence the assembler selection of the optimal financing scheme. Zhang et al. [31] made an operational decisions and financing strategies based on a closed-loop supply chain including in a financially constrained manufacturer and a retailer. Qian et al. [21] formulated a dynamic stochastic model to explore the coordination of operational and financial decisions of proportional investment agricultural marketing cooperatives. The study also identified the financial risks associated with uncertain market revenue and yield and the risk of redemption default. Yan et al. [29] focused on fresh agricultural products and analyzed the optimal operational and financing strategies for SMEs in different scenarios. The optimal decision is affected by various factors, such as financing rate, freshness effort cost coefficient, and the sensitivity coefficient of market freshness.

The second research stream is related to trade credit. Trade credit has addressed many financial problems when the bank financing is limited for some SMEs. Kouvelis and Zhao [15] argue that the retailer will always prefer manufacturer financing to bank financing, and the manufacturer will always finance the retailer. Chod 
[8] showed how debt financing distorts a retailer inventory decision and argued the mitigation of financing provided by a supplier. Zhou et al. [33] considered the ordering and payment issues for a retailer who enjoys the partial trade credit from suppliers and borrows funds from banks. Peura et al. [20] proved that trade credit can soften horizontal price competition in a Bertrand competition framework. Chen et al. [7] analyzed the efficacy of zero-interest early payment financing and positive-interest in-house factoring financing in a pull supply chain with a capital-constrained manufacturer who sells a product through a retailer with no capital constraints. Their results showed that the manufacturer production cost plays a significant role on the retailer preference on different financing schemes. Zhan et al. [30] studied the retailer advanced payment and reverse factoring models and then characterized their equilibrium solutions. Some researches focused on the green supply chain. An et al. [3] examined the impact of trade credit financing and green credit financing, respectively. Luo et al. [19] explored the optimal procurement decision in a two-echelon green supply chain considering two schemes, supplier and bank financing. Regardless of which scheme is used, the benefits of supply chain members are higher than those using the no-financing model. In addition, the retailer is a free rider in the green supply chain. From above analysis, the models in these two streams are not based on guarantor financing given their distinct focuses.

The third research stream is also related to the emerging practice of supply chain finance with guarantees. There are many guarantee mechanisms in supply chain finance. Tunca and Zhu [26] built a game-theoretical model and studied the role and efficiency of buyer intermediation in supplier financing based on the practice of JD, a large Chinese online retailer. Huang et al. [11] explored a buyer-backed purchase order financing scheme, wherein the buyer supports the supplier in financing through a loan guarantee. These two researches are presented from the perspective of retailer guarantee. Some researches also explore the effect of supplier guarantee. Jin et al. [12] studied the bank financing with supplier guarantee and they found that supplier guarantee may outperform bank financing with trade credit for the total supply chain profit. Zhou et al. [32] explored manufacturer guarantor financing in a four-party supply chain game. In addition, they also consider the guarantee from the third-party logistics. The members showed different preferences of leadership structures. Other guarantee schemes are also analyzed. Barach et al. [4] posited that money-back guarantees can be particularly effective at steering due to the alignment of buyer and platform interests, creating a good match. Different from these full guarantee schemes, partial guarantee becomes an other important guarantee schemes. Tang et al. [25] considered the use of partially guaranteed price contracts in an environment with price uncertainty, but no yield uncertainty. Luo et al. [18] used a real option approach and developed an investment and financing model with a partial guarantee. Yan et al. [28] and Lu et al. [17] designed a partial credit guarantee contract in the supply chain where a manufacture offers a credit guarantee for a capital-constrained retailer.

In order to distinguish our research question from previous literature, we have summarized some research paper which is revelent to our research in Table 1. As shown in Table 1, most researches study the financing equilibrium solutions under the newsvendor problem setting, which is a classical setting about market demand or yield uncertainty in supply chain finance. Moreover, most researches analyzed the bank financing scheme as a benchmark to compare with a new financing scheme. In recent years, some paper focused on guarantee financing scheme. The main difference is that we consider the effect of the value of the newsvendor's mortgage assets. In our paper, the value of assets has an impact on the newsvendor's equilibrium solution of order quantity. As depicted in Figure 1, if the value of assets is high enough, there is no bankruptcy risk for newsvendor's bank loan. Therefore, it is vital for a bank or a guarantor to concern the newsvendor's assets before making their own decisions in supply chain finance. Moreover, despite their partial examinations of the financing operation problem of capital constraints, most of the aforementioned studies take the core company perspective to discuss its role in supply chain finance. By contrast, the present study considers an innovative online financing scheme with guarantee. Generated by a real business, the optimal decision of the newsvendor on order quantity is examined in a capital-constrained supply chain with guarantee from the guarantor. We focused on different risk control mechanism generated by the guarantor to explore the optimal risk control strategy of the guarantor in different parameter settings. Therefore, the value and limitations of the cash deposit are examined are analyzed. The loan limit on the optimal decision of farmers and the profit of the governmental guarantee alliance are also studied. Moreover, two kinds of loan limit setting on the profit of the guarantor are also considered. 
TABLE 1. Contributions of author(s).

\begin{tabular}{|c|c|c|c|c|c|}
\hline Author(s) & $\begin{array}{l}\text { Newsvendor } \\
\text { problem }\end{array}$ & $\begin{array}{l}\text { Guarantee } \\
\text { policy }\end{array}$ & Assets & $\begin{array}{l}\text { Bank } \\
\text { financing }\end{array}$ & $\begin{array}{l}\text { Initial } \\
\text { capital }\end{array}$ \\
\hline Buzacott and Zhang [5] & $\checkmark$ & & $\checkmark$ & $\checkmark$ & $\checkmark$ \\
\hline Zhou et al. [33] & $\checkmark$ & & & $\checkmark$ & $\checkmark$ \\
\hline Luo et al. [19] & & & & $\checkmark$ & $\checkmark$ \\
\hline Kouvelis and Zhao [14] & $\checkmark$ & & & $\checkmark$ & $\checkmark$ \\
\hline An et al. [3] & & & & $\checkmark$ & $\checkmark$ \\
\hline Huang et al. [11] & $\checkmark$ & $\checkmark$ & & $\checkmark$ & $\checkmark$ \\
\hline Zhou et al. [32] & $\checkmark$ & $\checkmark$ & & $\checkmark$ & \\
\hline Chen et al. [6] & $\checkmark$ & & & $\checkmark$ & \\
\hline Chen et al. [7] & $\checkmark$ & & & $\checkmark$ & $\checkmark$ \\
\hline Sarkar and Saren [22] & & $\checkmark$ & & & \\
\hline Lu et al. [17] & $\checkmark$ & $\checkmark$ & & $\checkmark$ & $\checkmark$ \\
\hline Yan et al. [28] & $\checkmark$ & $\checkmark$ & & $\checkmark$ & $\checkmark$ \\
\hline Deng et al. $[9]$ & $\checkmark$ & & & $\checkmark$ & $\checkmark$ \\
\hline Chod [8] & $\checkmark$ & & & $\checkmark$ & \\
\hline Tunca and Zhu [26] & $\checkmark$ & $\checkmark$ & & $\checkmark$ & $\checkmark$ \\
\hline Khanna et al. [13] & & $\checkmark$ & & & \\
\hline Jin et al. [12] & $\checkmark$ & $\checkmark$ & & $\checkmark$ & $\checkmark$ \\
\hline Zhang et al. [31] & & & & $\checkmark$ & $\checkmark$ \\
\hline This paper & $\checkmark$ & $\checkmark$ & $\checkmark$ & $\checkmark$ & $\checkmark$ \\
\hline
\end{tabular}

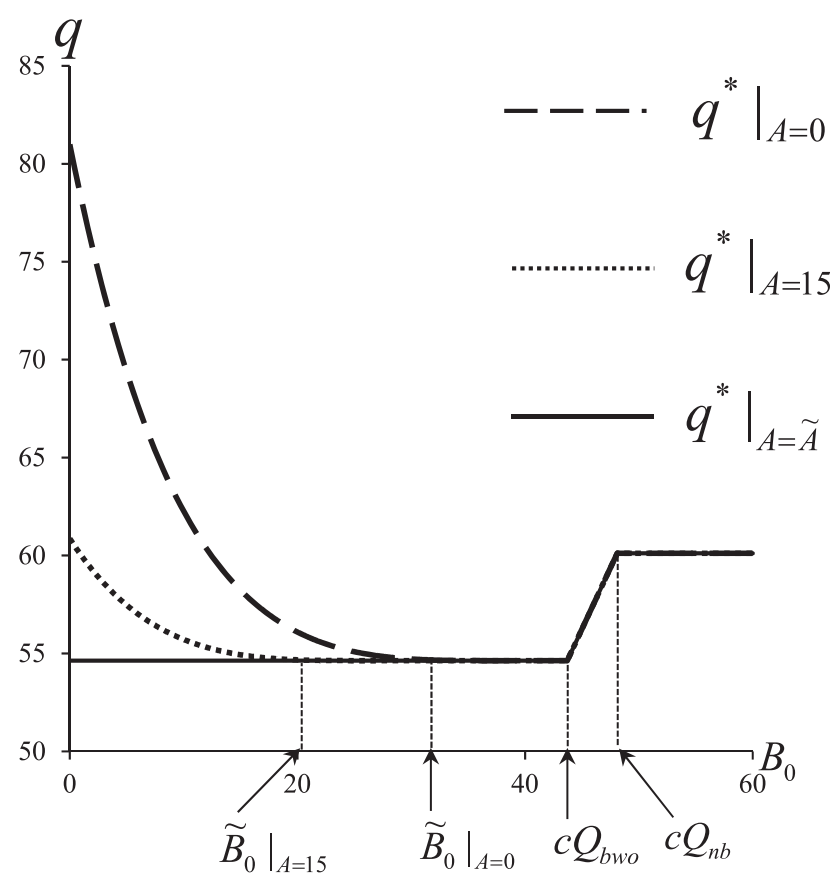

Figure 1. The combined impact of $A$ and $B_{0}$ on the newsvendor's order quantity. 
TABLE 2. List of parameters and notations.

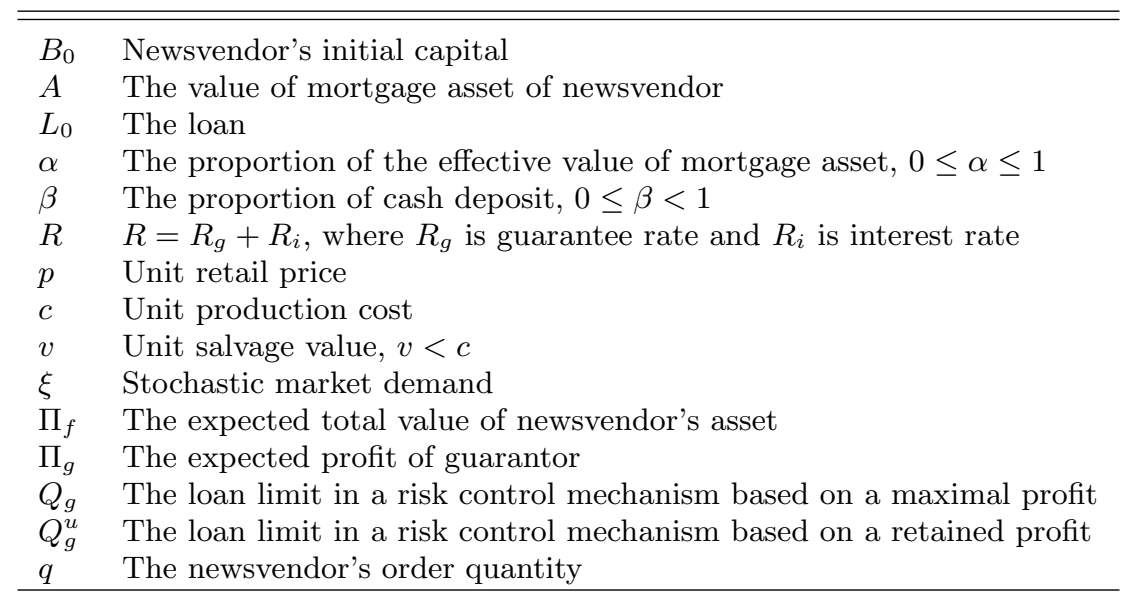

\section{MODELING FRAMEWORK}

This study considers a financing model in a supply chain comprising a capital-constrained small and mediumsized seller (here-after called "newsvendor"), bank and a third-party guarantee (here-after called "guarantor"). Before the the beginning of the sales season (time 0), the newsvendor has an initial capital $B_{0}$ and valuable asset $A$ that can be mortgaged to obtain loans. The newsvendor purchases products from suppliers and then sells to customers during the period $(0, T)$. Without sufficient cash to purchase, the newsvendor can borrow from the bank to avoid a supply chain scission. In the real business, due to their high risk and low asset, the newsvendor face difficulties to obtain bank loans. To eliminate this problem, the newsvendor can obtain support from the guarantor.

In time period $(0, T)$, the newsvendor faces an uncertain demand, which is denoted by a nonnegative random variable $\xi$. The probability density function is denoted as $f(\xi)$, and the cumulative distribution function is $F(\xi)$. $\bar{F}(\xi)=1-F(\xi)$. The hazard rate is defined as $h(\xi)=f(\xi) / \bar{F}(\xi)$. Let $H(\xi)=\xi h(\xi)$ denote the generalized failure rate. $F(\xi)$ is assumed as differentiable, strictly increasing, and absolutely continuous with $f(\xi)>0$ on $[0,+\infty)$. The demand distribution has a strictly increasing failure rate (IFR) property, that is, $h(\xi)$ is monotonously increasing in $\xi$. This condition is a commonly used assumption in supply chain modeling to determine the existence and uniqueness of an optimal solution. Many common distributions have IFR property, such as uniform, normal, exponential, and Gamma distribution [5]. The newsvendor faces the unit purchasing cost $c$. The selling price is assumed to be fixed $p$. At time 0 , the newsvendor decides on the order quantity $q$. The unit unsold products are assumed to have a salvage $v$, and $v<c$.

When $c q>B_{0}$, the newsvendor is capital-constrained and may borrows $L_{0}=c q-B_{0}$ from the bank using a guarantee. From business practice, to control the finance risk, the newsvendor needs to pay the bank with a cash deposit. In this study, the proportion of cash deposit is $\beta$, and $0 \leq \beta<1$. In the finance model with cash deposit, the newsvendor's actual loan is $(1-\beta) L$. To avoid the excessive finance, the equation $(1-\beta) L=\max \left\{0, L_{0}\right\}$ always holds. The guarantee fee rate is $R_{g}$ and bank interest rate is $R_{i}$, and $R=R_{g}+R_{i}$. Given the operational cost in the liquidation, when the value of the newsvendor's mortgaged asset is liquidated, the bank can only obtain a portion of the asset value. Specifically, the value of newsvendor's mortgaged asset will be reduced because the depreciation of some equipments. Therefore, we let $\alpha$ be the proportion of the effective assets and $\alpha \in[0,1]$. Table 2 shows the main parameters and notations. 


\section{Risk CONTROL MECHANism With CASH DEPOSIT}

\subsection{Optimal decision of the newsvendor}

In the risk control mechanism with cash deposit, at the end of sales season (Time $T$ ), the newsvendor's cash flow can be written as:

$$
B_{T}(\xi)=B_{0}+(p-v) \min \{\xi, q\}+v q-c q-\mathrm{RL},
$$

where $L=\frac{\max \left\{0, L_{0}\right\}}{1-\beta},(p-v) \min \{\xi, q\}+v q$ is the sale revenue, $c q$ is the total purchasing cost of all products, $\mathrm{RL}$ is the financing interest of the loan. Thus, the total value of newsvendor's assets is $\tilde{\Pi}_{f}(q, \xi)=B_{T}(\xi)+\alpha A$. If $\tilde{\Pi}_{f}(q, \xi)<0$, the newsvendor is bankrupt.

To clearly present the following results, we denote $\theta=1+\frac{R}{1-\beta}$ and define the following thresholds on order quantity and demand:

$$
\begin{aligned}
\hat{q} & =\frac{\theta B_{0}}{\theta c-v}, \quad \tilde{q}=\frac{\theta B_{0}+\alpha A}{\theta c-v}, \\
d_{1}(q) & =\frac{(\theta c-v)(q-\hat{q})}{p-v}, \quad d_{2}(q)=\frac{(\theta c-v)(q-\tilde{q})}{p-v} .
\end{aligned}
$$

To eliminate trivial cases, we assume $p>\theta c$.

Proposition 4.1. (1) If $B_{0} / c<q \leq \hat{q}$, the newsvendor has no asset liquidation risk; if $\hat{q} \leq q<\tilde{q}$, the newsvendor needs to initiate asset liquidation to pay off all debt; if $q>\tilde{q}$, the newsvendor has bankruptcy risk and the guarantor will repay the newsvendor's bad loan to the bank.

(2) If $\hat{q} \leq q<\tilde{q}$, for any demand $\xi$, the newsvendor has no bankruptcy risk but needs to initiate asset liquidation to pay off all debt. The total value of newsvendor's asset is $\tilde{\Pi}_{f}(q, \xi)=(p-v) \min \{\xi, q\}-(\theta c-v) q+\theta B_{0}+\alpha A$;

(3) If $q>\tilde{q}$, when demand $\xi<d_{2}(q)$, the newsvendor is bankrupt. The total value of newsvendor's asset is

$$
\tilde{\Pi}_{f}(q, \xi)= \begin{cases}(p-v)\left[\min \{\xi, q\}-d_{2}(q)\right], & \xi \geq d_{2}(q) ; \\ 0, & \xi<d_{2}(q) .\end{cases}
$$

Proposition 4.1 presents the relationship between the newsvendor's decision on order quantity and the bankruptcy risk. When the market demand is small and the newsvendor sets an excessive order quantity, the bankruptcy risk is high. From Proposition 4.1, at time $T$, four scenarios can be observed on the relationship among $B_{0}, B_{T}(\xi)$ and $\alpha A$ :

Case 1: $B_{0} \geq c q$. The order quantity $q$ satisfies $q \leq B_{0} / c$, which indicates that the newsvendor has sufficient initial capital to produce an optimal quantity and loan is not necessary.

Case 2: $B_{0}<c q, B_{T}(\xi) \geq 0$. The order quantity satisfies $B_{0} / c<q \leq \hat{q}$, which indicates that the newsvendor has sufficient capital to repay all debts.

Case 3: $B_{0}<c q,-\alpha A \leq B_{T}(\xi)<0$. The newsvendor's mortgage asset is liquidated to repay the debt, which indicates that the newsvendor does not have sufficient capital after the sales season.

Case 4: $B_{0}<c q, B_{T}(\xi)<-\alpha A$. The newsvendor is bankrupt and the guarantor repays the bad loan.

In Cases 2 and 3, the newsvendor has no bankruptcy risk and total assets can be presented as $\tilde{\Pi}_{f}(q, \xi)=$ $(p-v) \min \{\xi, q\}-(\theta c-v) q+\theta B_{0}+\alpha A$. Therefore, the expected total value of the newsvendor's assets is $\Pi_{f}(q)=\mathbb{E}_{\xi}\left(\tilde{\Pi}_{f}(q, \xi)\right)$, that is

$$
\Pi_{f}(q)= \begin{cases}(p-v)\left[q \bar{F}(q)+\int_{0}^{q} \xi f(\xi) \mathrm{d} \xi\right]-(c-v) q+B_{0}+\alpha A, & q \leq B_{0} / c ; \\ (p-v)\left[q \bar{F}(q)+\int_{0}^{q} \xi f(\xi) \mathrm{d} \xi\right]-(\theta c-v) q+\theta B_{0}+\alpha A, & B_{0} / c<q \leq \tilde{q} ; \\ (p-v)\left[q \bar{F}(q)-d_{2}(q) \bar{F}\left(d_{2}(q)\right)\right]+(p-v)\left[\int_{d_{2}(q)}^{q} \xi f(\xi) \mathrm{d} \xi\right], & q>\tilde{q} .\end{cases}
$$


Consider the case $q<B_{0} / c$, taking the derivative of $\Pi_{f}(q)$ with respect to $q$, we obtain $\mathrm{d} \Pi_{f}(q) / \mathrm{d} q=(p-v)$ $\bar{F}^{-1}(q)-(c-v)$. Thus, the optimal decision is $Q_{\mathrm{nb}}=\bar{F}^{-1}\left(\frac{c-v}{p-v}\right)$. Consider the situation where $B_{0} / c<q \leq \tilde{q}$, the optimal solution $Q_{\mathrm{bwo}}=\bar{F}^{-1}\left(\frac{\theta c-v}{p-v}\right)$ is similarly obtained. Consider the case $q>\tilde{q}$, in line with previous research [5], we obtain

$$
\frac{\mathrm{d}^{2} \Pi_{f}(q)}{\mathrm{d} q^{2}}=-(p-v) \bar{F}(q)\left[h(q)-\frac{(\theta c-v)}{(p-v)} h\left(d_{2}(q)\right)\right] .
$$

Given that the demand has IFR property, and $d_{2}(q)<q$, we obtain $\frac{\mathrm{d}^{2} \Pi_{f}(q)}{\mathrm{d} q^{2}}<0$. Therefore, $\Pi_{f}(q)$ is strictly concave on $q$. The optimal solution $Q_{\text {bwr }}$ satisfies:

$$
\bar{F}\left(Q_{\mathrm{bwr}}\right)=\frac{\theta c-v}{p-v} \bar{F}\left(d_{2}\left(Q_{\mathrm{bwr}}\right)\right) .
$$

Let $\tilde{B}_{0}=\frac{\theta c-v}{\theta} Q_{\mathrm{bwo}}-\frac{\alpha A}{\theta}, \bar{B}_{0}=\frac{(\theta c-v) Q_{\mathrm{bwo}}}{\theta}, \tilde{A}=\frac{(\theta c-v) Q_{\mathrm{bwo}}}{\alpha}, \bar{A}=\frac{(\theta c-v) Q_{\mathrm{bwo}}-\theta B_{0}}{\alpha}$, we get the optimal quantity decision.

Proposition 4.2. (1) If $B_{0}>c Q_{\mathrm{nb}}$, then the optimal order quantity is $q^{*}=Q_{\mathrm{nb}}$.

(2) If $c Q_{\mathrm{bwo}} \leq B_{0} \leq c Q_{\mathrm{nb}}$, then the newsvendor spends all initial capital to purchase, that is, $q^{*}=B_{0} / c$.

(3) If the asset $A<\tilde{A}$, then: when $0 \leq B_{0} \leq \tilde{B}_{0}, q^{*}=Q_{\text {bwr }}$; when $\tilde{B}_{0} \leq B_{0} \leq c Q_{\text {bwo }}$, $q^{*}=Q_{\text {bwo }}$.

(4) If the asset $A \geq \tilde{A}$, then, for all $0 \leq B_{0} \leq c Q_{\mathrm{bwo}}$, there is $q^{*}=Q_{\mathrm{bwo}}$.

Proposition 4.2 shows that the optimal quantity $q^{*}$ is segmented and correlated with the initial budget $B_{0}$ and mortgage asset $A$. In the following corollary, we further investigate how the two parameters, $B_{0}$ and $A$, affect the optimal quantity decisions.

Corollary 4.3. (i) If $A<\tilde{A}$ and $0 \leq B_{0}<\tilde{B}_{0}$, the newsvendor's optimal solution $Q_{\mathrm{bwr}}$ is decreasing in $B_{0}$ and $A$; (ii) If $A \geq \tilde{A}$, the newsvendor's optimal solution $Q_{\mathrm{bwo}}$ is independent with $B_{0}$ and $A$, and the newsvendor have no bankruptcy risk for any $B_{0}$.

A numerical study is carried out to show the combined impact of newsvendor's initial capital and mortgage assets on the optimal quantity decision. The uncertain demand is assumed to follow a Gamma distribution with parameters $\mu=100, \sigma / \mu=0.5$ and the supply chain parameters are: $p=1, c=0.8, v=0.1, R=0.05, \alpha=0.8$. Figure 1 illustrates that a high value of mortgage asset reduces the optimal solution. With less mortgage assets, the newsvendor borrows a higher amount from the financing platform to produce more products. Thus, the bank may face the huge bankruptcy risk of the newsvendor.

\subsection{Guarantor's quantity preference}

Next, the guarantor's profit is examined. From Proposition 4.1, the guarantor has a risk of repayment if and only if $q>\tilde{q}$. Thus, the profit functions of guarantor is determined below.

Corollary 4.4. (i) If $q \leq B_{0} / c$, the guarantor has no profit and no risk of repayment.

(ii) If $B_{0} / c<q \leq \tilde{q}$, the profit of the guarantor is $R_{g}\left(c q-B_{0}\right) /(1-\beta)$, and the guarantor has no risk of repayment.

(iii) If $q>\tilde{q}$, the guarantor has no risk of repayment when $\xi>d_{2}(q)$, otherwise, the guarantor has risk of repayment. The profit function of the guarantor is

$$
\tilde{\Pi}_{g}(q, \xi)= \begin{cases}\frac{R_{g}}{1-\beta}\left(c q-B_{0}\right), & \xi>d_{2}(q) \\ \frac{R_{g}}{1-\beta}\left(c q-B_{0}\right)-(p-v)\left[d_{2}(q)-\xi\right], & \xi \leq d_{2}(q) .\end{cases}
$$


Based on Corollary 4.4, the expected profit of guarantor can be written as

$$
\Pi_{g}(q)= \begin{cases}0, & q \leq B_{0} / c ; \\ \frac{R_{g}}{1-\beta}\left(c q-B_{0}\right), & B_{0} / c<q \leq \tilde{q} ; \\ \frac{R_{g}}{1-\beta}\left(c q-B_{0}\right)-(p-v) d_{2}(q) F\left(d_{2}(q)\right)+(p-v) \int_{0}^{d_{2}(q)} F(\xi) \mathrm{d} \xi, & q>\tilde{q} .\end{cases}
$$

Clearly, when $q>\tilde{q}$, the optimal decision for the newsvendor on $q$ is not necessarily the best solution for the guarantor. Easily, $\Pi_{g}(q)$ is strictly concave on $q$. Thus, from the first-order condition of $\Pi_{g}(q)$, the optimal preference of guarantor, $Q_{g}=\tilde{q}+q_{g}$ is obtained, where

$$
q_{g}=\frac{p-v}{\theta c-v} F^{-1}\left(\frac{R_{g} c}{(1-\beta)(\theta c-v)}\right) .
$$

\subsection{Effect of risk control with cash deposit}

After comparing the optimal quantity preferences between the guarantor and the newsvendor, we have the following proposition. We denote a threshold $A_{0}^{*}=\frac{\theta c-v}{\alpha}\left[\bar{F}^{-1}\left(\frac{\theta c-v}{p-v} \bar{F}\left(\frac{\theta c-v}{p-v} q_{g}\right)\right)-q_{g}\right]$.

Proposition 4.5. (1) When $0 \leq A<A_{0}^{*}$, there exists $B_{0}^{*} \in\left[0, \tilde{B}_{0}\right]$, such that: if $0 \leq B_{0} \leq B_{0}^{*}$, then $Q_{g}<Q_{\mathrm{bwr}}$, and if $B_{0}^{*} \leq B_{0} \leq \tilde{B}_{0}$, then $Q_{g} \geq Q_{\mathrm{bwr}}$.

(2) When $A \geq A_{0}^{*}, Q_{g} \geq Q_{\mathrm{bwr}}$ holds for any $B_{0} \in\left[0, \tilde{B}_{0}\right]$.

(3) For any $A, Q_{g}>Q_{\text {bwo }}$ holds for $B_{0} \geq \tilde{B}_{0}$.

Proposition 4.5 reveals that the optimal solution of the guarantor may be lower than that of the newsvendor, which depends on the assets and initial capital of the latter. A newsvendor with less initial capital or asset has high bankruptcy risk. Thus, the guarantor needs to construct a risk control mechanism. Furthermore, the property of the guarantor profit is examined in the following corollary.

Corollary 4.6. If $0<B_{0}<B_{0}^{*}$, then $\Pi_{g}$ is increasing in $A$, where $0 \leq A<A_{0}^{*}$.

Corollary 4.6 shows that the guarantor can obtain greater profit by guaranteeing a newsvendor with more asset and bankruptcy risk. However, when the newsvendor has no bankruptcy risk, the guarantor profit shows other properties. Given $A$, if $\tilde{B}_{0}<B_{0}<c Q_{\mathrm{bwo}}$, the newsvendor has no bankruptcy risk and the guarantor profit decreases in $B_{0}$. When $B_{0} \in\left[\bar{B}_{0}, c Q_{\mathrm{bwo}}\right]$, the guarantor has no risk of repayment. Therefore, when $B_{0} \in\left[\tilde{B}_{0}, \bar{B}_{0}\right]$, a bankruptcy risk for the newsvendor remains and the guarantor profit depends on the value of the newsvendor's mortgage assets. If and only if $B_{0}>\bar{B}_{0}$, regardless of the mortgage assets that the newsvendor owns, the guarantor has no risk.

Figure 1 also indicates that a capital-constrained newsvendor may make aggressive decisions about order quantity, which can lead to a huge risk of repayment for the guarantor. Thus, we further study how risk control mechanisms can reduce the risk caused by excessive financing of lending companies with small initial capital.

In practice, the guarantor adopts measures to compensate for the risk of repaying bad loans, typically charging a certain proportion of the loan amount as cash deposit. Next, we explore the impact of the cash deposit on the newsvendor's decision and the guarantor profit. In order to clarify the expression, $Q_{\mathrm{bwo}}^{0}$ and $Q_{\mathrm{bwr}}^{0}$ denote the newsvendor's optimal solution $Q_{\mathrm{bwo}}, Q_{\mathrm{bwr}}$ when $\beta=0$.

Lemma 4.7. When $B_{0} \leq c Q_{\mathrm{bwo}}, q^{*}$ is decreasing in $\beta$.

Lemma 4.7 reveals that the proportion of cash deposit has a negative effect on the quantity decision of newsvendors with low initial capital. In this case, the cash deposit is an effective solution of the guarantor to reduce the bankruptcy risk of newsvendors. Next, we define $B_{0}^{\beta}$ such that: $B_{0}^{\beta}$ satisfies $\frac{R_{g}}{R}=F\left(d_{2}\left(Q_{\mathrm{bwr}}\right)\right)$ when $\frac{R_{g}}{R}<F\left(d_{2}\left(Q_{\mathrm{bwr}}\right)\right)$, and $B_{0}^{\beta}=0$ when $\frac{R_{g}}{R} \geq F\left(d_{2}\left(Q_{\mathrm{bwr}}\right)\right)$. The following proposition considers the impact of cash deposit on the guarantor profit. 


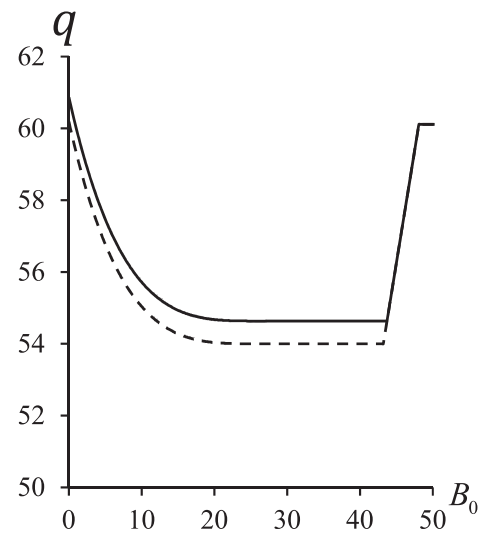

(a)

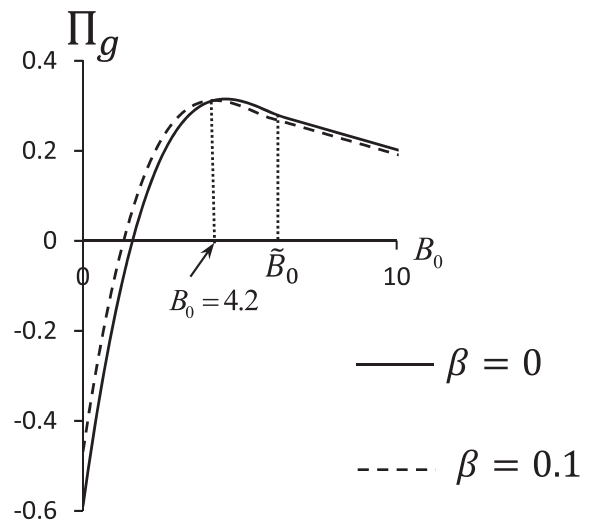

(b)

Figure 2. The impact of case deposit on the newsvendor's order quantity and the guarantor's profit. (a) $\frac{\sigma}{\mu}=0.7$. (b) $\frac{\sigma}{\mu}=0.9$.

Proposition 4.8. Comparing with the case without cash deposit,

(1) when $A<\tilde{A}$ and $\tilde{B}_{0} \leq B_{0} \leq c Q_{\mathrm{bwo}}$ or $A>\tilde{A}$ and $0 \leq B_{0}<c Q_{\mathrm{bwo}}$, the risk control mechanism with cash deposit will reduce the profit of the guarantor;

(2) when $0 \leq A<A_{0}^{*}$ and $B_{0}^{\beta} \leq B_{0}<B_{0}^{*}$, the risk control mechanism with cash deposit will improve the profit of the guarantor and the guarantor's profit is increasing in $\beta$.

Proposition 4.8(1) shows that, when the newsvendor has no bankruptcy risk, the guarantor profit decreases with the adoption of the risk control mechanism with cash deposit, because the newsvendor reduces the order quantity. Proposition 4.8(2) illustrates that, when the newsvendor has a bankruptcy risk, the guarantor profit increases because the cash deposit can cover part of the risk loss.

Assume that the stochastic demand $\xi$ follows a Gamma distribution with expectation $\mu=100, \sigma / \mu=0.7$ (or $0.9), p=1, c=0.8, v=0.1, R=0.05, \alpha=0.8$ and $A=15$, the effects of case deposit on the order quantity and the guarantor profit are plotted in Figures $2 \mathrm{a}$ and $2 \mathrm{~b}$ separately. As shown in Figure 2b, on the one hand, when the newsvendor has low initial capital, adopting the risk control mechanism with cash deposit can increase the guarantor profit. On the other hand, when the newsvendor has high initial capital, the guarantor can obtain high profits without adopting the risk control mechanism with cash deposit. The reason is that, at a high initial capital, without the risk control mechanism, the quantity decision of the newsvendor does not increase the repayment risk for the guarantor. Thus, the use of risk control mechanism reduces the quantity decision of a newsvendor and reduces the guarantor profit.

\section{RiSK CONTROL MECHANISMS WITH LOAN LIMIT}

\subsection{Two risk control mechanisms with loan limit}

The aforementioned analysis reveals that the newsvendor reduces the quantity decisions in the risk control mechanism with cash deposit. However, when the newsvendor has low initial capital, the quantity decision of the newsvendor remains large. Consequently, the guarantor faces a huge risk of bankruptcy. In practice, popular risk control mechanisms point out that the guarantor may set a loan limit on financing capital-constrained newsvendors. Note that when considering the risk control mechanism with loan limit, the cash deposit is not used, that is, $\beta=0$. 
This section considers a financing limit setting model, in which the guarantor proposes a loan limit such that the product quantity of the newsvendor is not greater than $Q_{1 \mathrm{mt}}$. Thus, the upper bound of loan limit is $c Q_{\mathrm{lmt}}-B_{0}$. In line with real business, the following two types of loan limits are examined.

First, the upper bound of loan limit is set by a maximal profit. If the guarantor proposes a loan limit by maximizing its own profit, then $Q_{\mathrm{lmt}}=Q_{g}$. From Proposition 4.5, when $0 \leq A<A_{0}^{*}$ and $0 \leq B_{0} \leq B_{0}^{*}$, the newsvendor quantity decision is limited by $Q_{g}$. When $0 \leq A<A_{0}^{*}$ and $B_{0}^{*} \leq B_{0} \leq \tilde{B}_{0}$ (or $A \geq A_{0}^{*}$ and $\left.B_{0} \in\left[0, \tilde{B}_{0}\right]\right)$, the loan limit has no effect on the decision of the newsvendor, who can then obtain the optimal solution $Q_{\mathrm{bwr}}$. Thus, when the newsvendor quantity decision is $Q_{g}$, which is less than the newsvendor optimal quantity, the guarantor can obtain a higher profit.

Corollary 5.1. The quantity $Q_{g}$ is increasing in $B_{0}$.

Corollary 5.1 indicates that the guarantor allows more loans to newsvendors with high capital, who face lower risk of being bankrupt than those with low initial capital.

Second, the upper bound of loan limit is set by a retained profit. Proposition 4.5 shows that the newsvendor's optimal quantity can be larger than $Q_{g}$. To improve the newsvendor's loan amount and control the repayment risk of the guarantor, the risk control mechanism is set as the upper bound of loan limit by a retained profit, which is only related to the newsvendor's loan amount and a retained return rate $R_{f}$. The retained profit is assumed to be not larger than the maximized expected profit, that is, $\Pi_{g}\left(Q_{g}\right)=R_{f}\left(c Q_{g}-B_{0}\right)$.

When $q>Q_{g}, \frac{\mathrm{d} \Pi(q)}{\mathrm{d} q}=-(c-v) F\left(d_{2}(q)\right)-\frac{R_{i}}{1-\beta} c F\left(d_{2}(q)\right)<0$, the guarantor profit is increasing in $q$. Therefore, an unique threshold $Q_{g}^{u}$ must exist, which is larger than $Q_{g}$ and satisfies that $\Pi_{g}\left(Q_{g}^{u}\right)=R_{f}\left(c Q_{g}^{u}-B_{0}\right)$, that is,

$$
R_{g}\left(c Q_{g}^{u}-B_{0}\right)-(p-v) d_{2}\left(Q_{g}^{u}\right) F\left(d_{2}\left(Q_{g}^{u}\right)\right)+(p-v) \int_{0}^{d_{2}\left(Q_{g}^{u}\right)} \xi f(\xi) \mathrm{d} \xi=R_{f}\left(c Q_{g}^{u}-B_{0}\right) .
$$

Given the complexity of solving $Q_{g}^{u}$ in equation (5.1), we present a numerical example. Set $\xi$ follows a Gamma distribution with parameters $\mu=100, \sigma / \mu=0.5$. Let $p=1, c=0.8, v=0.1, R=0.05$ and $\alpha=0.8$. Figure 3 depicts the effect of newsvendor's initial capital on the loan limit set by the guarantor. Figure 3 shows a point $\tilde{B}_{0}^{*}$ when the initial capital $B_{0}$ of the financing enterprise satisfies: when $B_{0}<\tilde{B}_{0}^{*}$, then $q^{*}>Q_{g}^{u}$; when $B_{0}>\tilde{B}_{0}^{*}$, then $q^{*}<Q_{g}^{u}$. The following Corollary 5.2 shows the trend of $Q_{g}^{u}$ with $B_{0}$.

Corollary 5.2. The quantity $Q_{g}^{u}$ is increasing in $B_{0}$.

Combined with Corollaries 5.1 and 5.2, $Q_{g}^{u}$ has the similar property with $Q_{g}$. Consequently, if the guarantor reduces its own risk by setting a loan limit, both two mechanisms allow a newsvendor with high initial capital to obtain more loans. Figure 3 depicts an example, when the initial capital is small, the optimal decision of the newsvendor is larger than $Q_{g}$.

\subsection{Comparison between two kinds of risk control mechanisms with loan limit}

From the aforementioned discussion, setting the upper bound of loan limit can reduce the excessive quantity of the newsvendor. Compared with that set by a retained profit, the loan limit that is set by a maximal profit is smaller and thus the newsvendor's loan amount is evidently reduced in real business. The low loan limit reduces the newsvendor's willingness to obtain financing and may thus be detrimental to the entire supply chain. If the newsvendor's quantity $q^{*}$ such that $Q_{g} \leq q^{*} \leq Q_{g}^{u}$, then the guarantor can not only ensure its own retained profit but also make the newsvendor's order quantity be greater than $Q_{g}$. Thus, the loan limit that is set by a retained profit is more attractive to the newsvendor. The related property of guarantor under the two risk control mechanisms with loan amount is analyzed in the following proposition.

Proposition 5.3. (1) When the upper bound of loan limit is $Q_{g}$, $\Pi_{g}^{*}$ is increasing in $B_{0}$ with $B_{0}<B_{0}^{*}$;

(2) When the upper bound of loan limit is $Q_{g}^{u}$, $\Pi_{g}^{*}$ is increasing in $B_{0}$ with $B_{0}<\tilde{B}_{0}^{*}$. 


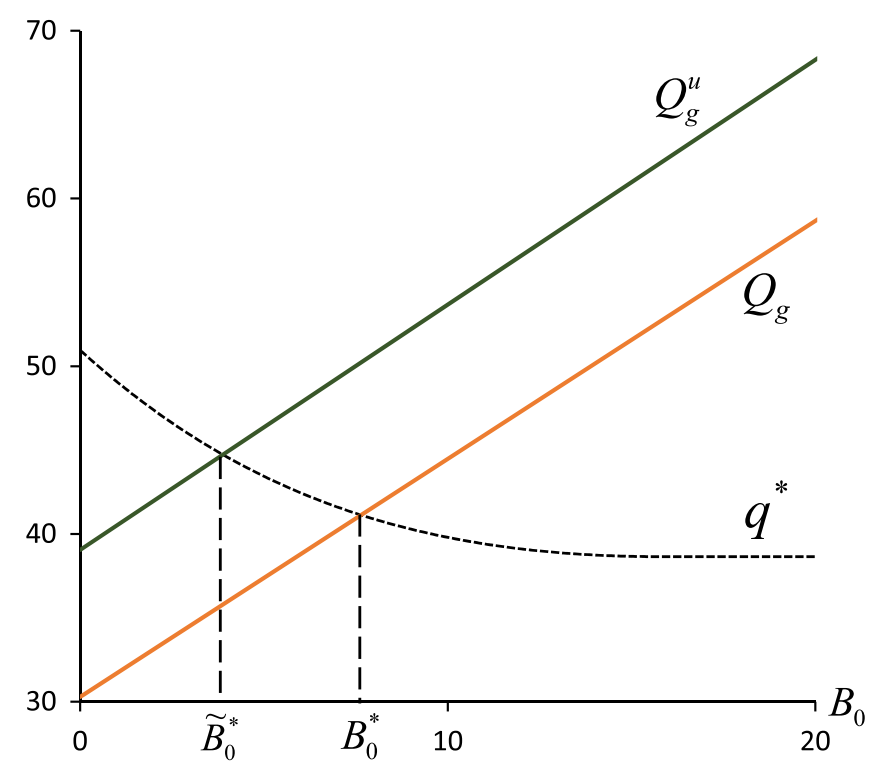

Figure 3 . The impact of $B_{0}$ on the loan limit.

Combined with Proposition 5.3 and Figure 3, the newsvendor makes varying quantity decisions under different scenarios according to $B_{0}$. When the upper bound of the loan limit is $Q_{g}$, the quantity decision of newsvendor with low initial capital $\left(0 \leq B_{0}<B_{0}^{*}\right)$ is affected. Therefore, when $0 \leq B_{0}<B_{0}^{*}$, the newsvendor has a huge risk of bankruptcy. Thus, the guarantor profit is $\Pi_{g}\left(B_{0}\right)=R_{g}\left(c Q_{g}-B_{0}\right)-(p-v) d_{2}\left(Q_{g}\right) F\left(d_{2}\left(Q_{g}\right)\right)+$ $(p-v) \int_{0}^{d_{2}\left(Q_{g}\right)} \xi f(\xi) \mathrm{d} \xi ;$ when $B_{0}^{*} \leq B_{0}<\tilde{B}_{0}, \Pi_{g}\left(B_{0}\right)=R_{g}\left(c Q_{\mathrm{bwr}}-B_{0}\right)-(p-v) d_{2}\left(Q_{\mathrm{bwr}}\right) F\left(d_{2}\left(Q_{\mathrm{bwr}}\right)\right)+$ $(p-v) \int_{0}^{d_{2}\left(Q_{\mathrm{bwr}}\right)} \xi f(\xi) \mathrm{d} \xi$; when $\tilde{B}_{0} \leq B_{0}<c Q_{\mathrm{bwo}}, \Pi_{g}\left(B_{0}\right)=\left(c Q_{\mathrm{bwo}}-B_{0}\right) R_{g}$; when $B_{0} \geq c Q_{\mathrm{bwo}}$, the newsvendor does not need to loan and thus $\Pi_{g}\left(B_{0}\right)=0$. When $A \geq \tilde{A}$, the newsvendor has no bankruptcy risk, and therefore, when $0 \leq B_{0}<B_{0}^{*}$, the newsvendor can make an order quantity decision that is larger than $Q_{g}$. In this study, only the situation where $A<\tilde{A}$ is considered.

Similarly, next we focus on the risk control mechanism with limit loan $Q_{g}^{u}$. If $0 \leq B_{0}<\tilde{B}_{0}^{*}$, then $\Pi_{g}\left(B_{0}\right)=R_{g}\left(c Q_{g}^{u}-B_{0}\right)-(p-v) d_{2}\left(Q_{g}^{u}\right) F\left(d_{2}\left(Q_{g}^{u}\right)\right)+(p-v) \int_{0}^{d_{2}\left(Q_{g}^{u}\right)} \xi f(\xi) \mathrm{d} \xi ;$ if $\tilde{B}_{0}^{*} \leq B_{0}<\tilde{B}_{0}$, $\Pi_{g}\left(B_{0}\right)=R_{g}\left(c Q_{\mathrm{bwr}}-B_{0}\right)-(p-v) d_{2}\left(Q_{\mathrm{bwr}}\right) F\left(d_{2}\left(Q_{\mathrm{bwr}}\right)\right)+(p-v) \int_{0}^{d_{2}\left(Q_{\mathrm{bwr}}\right)} \xi f(\xi) \mathrm{d} \xi ;$ if $\tilde{B}_{0} \leq B_{0}<c Q_{\mathrm{bwo}}$, $\Pi_{g}\left(B_{0}\right)=\left(c Q_{\mathrm{bwo}}-B_{0}\right) R_{g}$; if $B_{0} \geq c Q_{\mathrm{bwo}}, \Pi_{g}\left(B_{0}\right)=0$. If the loam limit is not existed, when $0 \leq B_{0}<\tilde{B}_{0}$, $\Pi_{g}\left(B_{0}\right)=R_{g}\left(c Q_{\mathrm{bwr}}-B_{0}\right)-(p-v) d_{2}\left(Q_{\mathrm{bwr}}\right) F\left(d_{2}\left(Q_{\mathrm{bwr}}\right)\right)+(p-v) \int_{0}^{d_{2}\left(Q_{\mathrm{bwr}}\right)} \xi f(\xi) \mathrm{d} \xi ;$ when $\tilde{B}_{0} \leq B_{0}<c Q_{\mathrm{bwo}}$, $\Pi_{g}\left(B_{0}\right)=\left(c Q_{\mathrm{bwo}}-B_{0}\right) R_{g}$; if $B_{0} \geq c Q_{\mathrm{bwo}}, \Pi_{g}\left(B_{0}\right)=0$.

Figure 4 depicts the relationship between the guarantor profit and the initial capital of the newsvendor under two different market risks (the greater $\sigma / \mu$ represents a higher market risk). In the figure, stochastic demand $\xi$ follows a Gamma distribution with parameters $\mu=100, \sigma / \mu=0.7$ (or 0.9 ), $p=1, c=0.8, v=0.1, R=0.05$, $\alpha=0.8$. Figure 4 shows that without a loan limit, the guarantor profit may be negative. With the loan limit, the guarantor profit is always positive. Figure 4 also shows that, if the guarantor sets $Q_{g}^{u}$ as loan limit, then the profit is lower than the loan limit $Q_{g}$. However, choosing $Q_{g}^{u}$ as loan limit can satisfy the newsvendor's preference on high quantity, which can strengthen their willingness to obtain loans for production. Simultaneously, the guarantor profit is similarly high. Therefore, the loan limit of $Q_{g}^{u}$ has practical significance. Figure 4 also shows that with the increase of market risk, the guarantor profit decreases. 


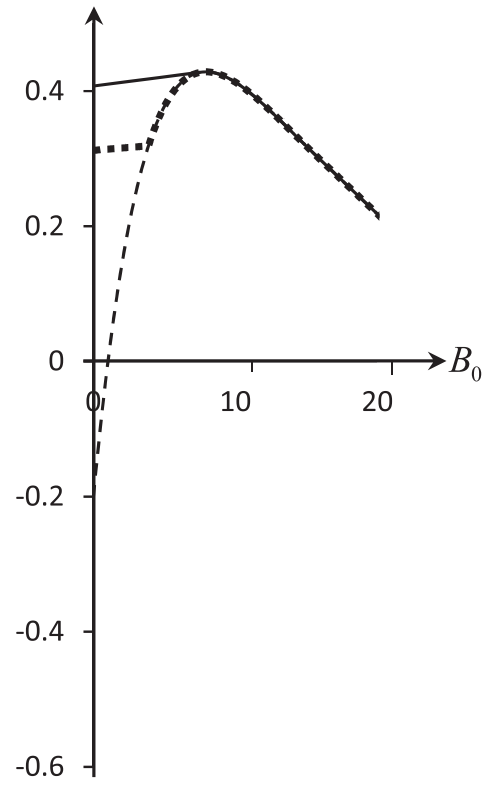

(a)

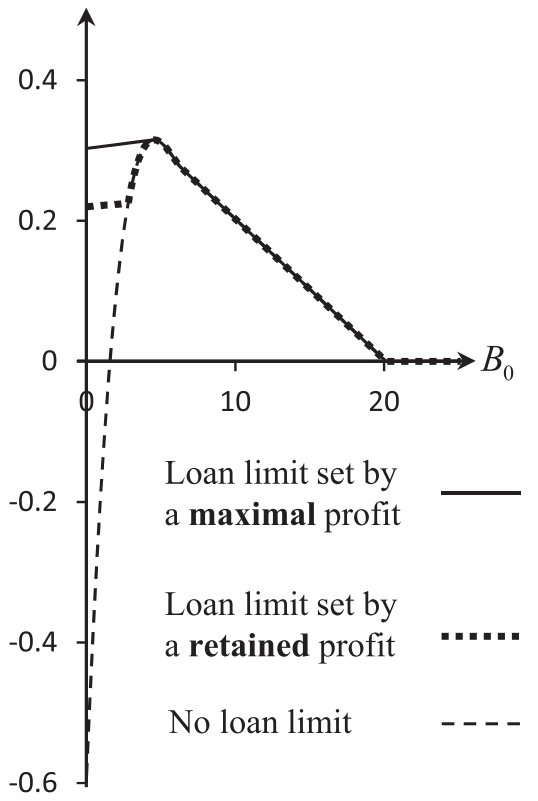

(b)

Figure 4. The impact of loan limit on the the profit of the guarantor. (a) $\frac{\sigma}{\mu}=0.7$. (b) $\frac{\sigma}{\mu}=0.9$.

Proposition 5.4. (1) In the risk control mechanism with loan limit $Q_{g}$, when $0<B_{0}<B_{0}^{*}$, the probability of being bankruptcy for the newsvendor is independent of $B_{0}$; when $B_{0}^{*}<B_{0}<\tilde{B}_{0}$, the probability of being bankruptcy for the newsvendor is decreasing in $B_{0}$;

(2) In the risk control mechanism with loan limit $Q_{g}^{u}$, when $0<B_{0}<\tilde{B}_{0}^{*}$, the probability of being bankruptcy for the newsvendor is higher than that in the mechanism with the loan limit $Q_{g}$.

Proposition 5.4 presents that the newsvendor's initial capital has a significant effect on the newsvendor's bankruptcy risk. When the newsvendor has low initial capital, the loan limit can evidently reduce the bankruptcy risk. Under the two risk control mechanisms with loan limits, the risk of newsvendors with low initial capital becomes constant. With the low initial capital, these newsvendors cannot obtain the optimal loans to make the best decision on quantity.

Setting the same parameters in Figure 4, Figure 5 depicts the relationship between the bankruptcy risk and the initial capital of the newsvendor under two different market cases. Clearly, the loan limit can effectively reduce the risk of bankruptcy for a newsvendor with a low initial capital. With a loan limit, the guarantor effectively reduces the newsvendor's quantity decision and therefore its bankruptcy risk. Figure 5 also analyzes the probability of the newsvendor's bankruptcy with different loan limits. The guarantor allows the newsvendor to make a quantity decision that is larger than $Q_{g}$ when a retained profit can be obtained. At this time, the bankruptcy risk is higher when the loan limit is $Q_{g}^{u}$ than when the loan limit $Q_{g}$, but still extremely lower than that without a loan limit. Therefore, this loan limit for risk management mechanism has strong practical significance and application value.

\section{Numerical Simulation AND ANALYSis}

This section analyzes the impact of cash deposit and loan limit on the rate of bad loan for the financing platform and the total guarantor profit. The numerical study has the following parameters: $p=1, v=0$, 


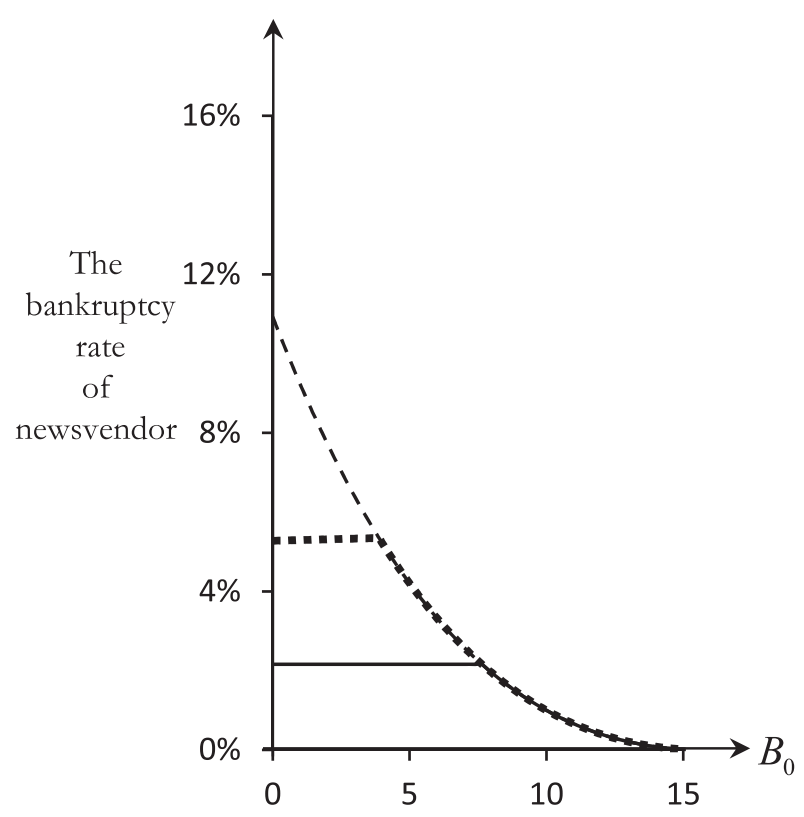

(a)

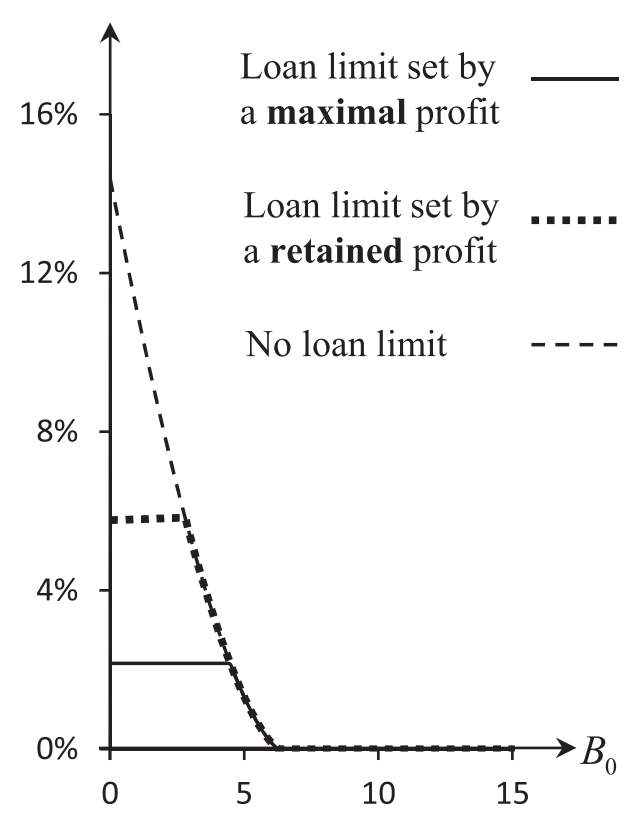

(b)

FigURE 5. The impact of loan limit on the probability of newsvendor's bankruptcy. (a) $\frac{\sigma}{\mu}=0.7$. (b) $\frac{\sigma}{\mu}=0.9$.

$\alpha=0.8, R=0.05, R_{g}=0.02, R_{f}=0.01$. Market demand is assumed to follow a Gamma distribution and the probability density function is $f(x)=\frac{x^{\tau-1} e^{x / \nu}}{\nu^{\tau} \Gamma(\tau)}, \tau=\left(\frac{1}{\sigma / \mu}\right)^{2}, \nu=\frac{\mu}{\tau}$. Through a uniform distribution, $\mu$ is generated randomly with [50,150], while $\sigma / \mu$ is generated randomly with $[0.2,0.95]$. For each example, the actual market demand $\xi$ is randomly evaluated according to the distribution of demand after its function is obtained. Simultaneously, through a uniform distribution, $B_{0}$ is assumed to be generated randomly with $[0,50]$ and $A$ is generated randomly with $[0,20] . c$ ranges from $\left[0.5, \frac{1}{1+R}\right]$ with the uniform distribution. Note that $\beta=0$ is considered in the case with loan limit, and $\beta=0.1$ in the case with the cash deposit.

\subsection{Impact of loan limit on bad loan rate}

Figure 6 shows the bad loan rate changes of the financing platform under 10 random simulations. When $B_{T}(\xi)+\alpha A<0$, the newsvendor is considered bankrupt. Each simulation randomly generates 100 sets of data and simulates the bankruptcy of several newsvendors under different circumstances through varying initial capital, mortgage assets, production cost, and market risk and demand.

Figure 6a shows that without a loan limit, the rate of bad loan for the financing platform is relatively high. From 10 simulations, the curves on the rate of bad loan without loan limit are above the two curves with loan limits. The possible reason is that when without a loan limit, several newsvendors are likely to seek too much production loan, finally cannot pay, and become bankrupt. With a loan limit, such excessive loan can be effectively controlled, and the bankruptcy risk for the newsvendor can be greatly reduced, thereby reducing the rate of bad loan for the financing platform. In this regard, the loan limit set by a maximal profit can be most effective. 


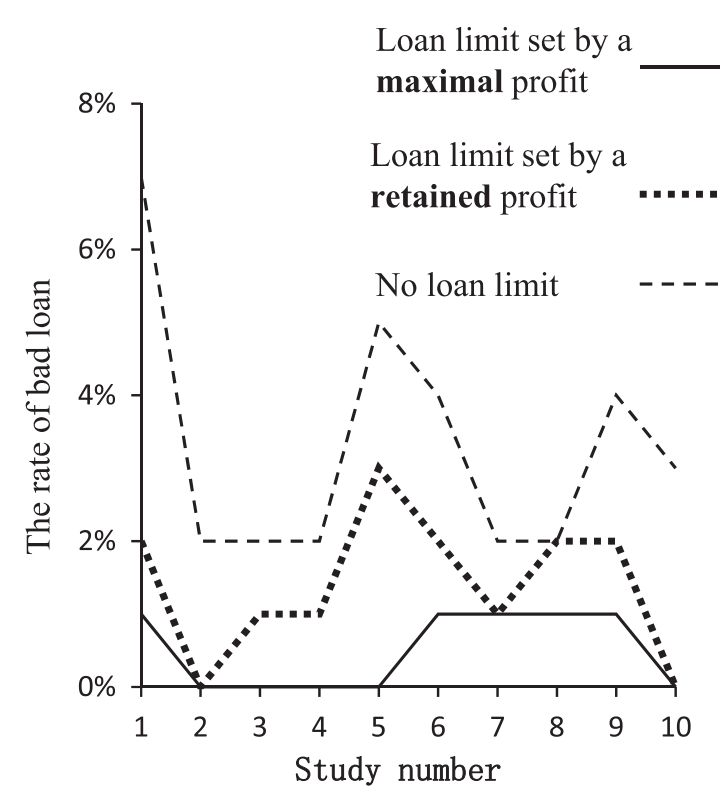

(a)

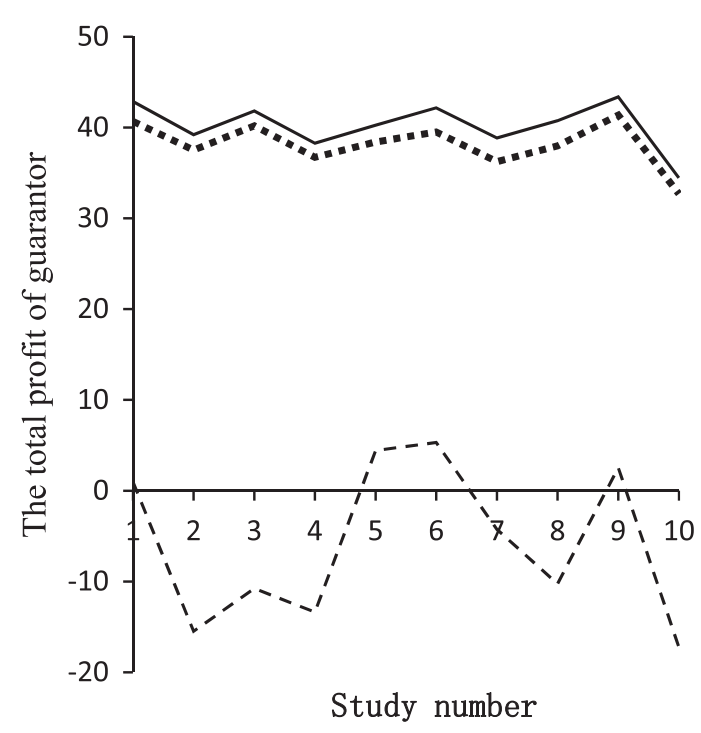

(b)

Figure 6 . The impact of security deposit on the bad debt rate of financing platform and the total income of guarantor.

\subsection{Impact of loan limit on the guarantor profit}

Next, we analyze the effect of loan limit on the total guarantor profit. The parameters in the simulation are the same as in the previous example. Figure $6 \mathrm{~b}$ shows the changes of total guarantor profit under 10 random simulations. Simultaneously, 100 sets of data in each simulation represent the guarantee for 100 newsvendor loans. Figure $6 \mathrm{~b}$ shows that without a loan limit, the expected guarantor profit is negative, because the guarantor bears a huge risk of repayment due to excessive loan of several newsvendors. Even with a positive expected profit, the value is far less than that with loan limit. The expected guarantor profit is always positive with a loan limit, due to governmental guarantee.

Among the two kinds risk control mechanisms, guarantee enterprises are more inclined to choose the financing scheme with the loan limit that is set according to their maximal profit. However, the above analysis shows that, when the newsvendor has low initial capital, their order quantity is greatly reduced by the loan limit, which in turn greatly reduces their willingness to obtain financing. Compared with the loan limit that is set by a maximal guarantor profit, the loan limit that is set by a retained profit can allow the newsvendor with low initial capital to increase order quantity to obtain a higher loan amount than that set by a maximal profit. Financing is therefore more attractive to newsvendors, while the expected guarantor profits are ensured as always positive.

\subsection{Impact of cash deposit on the bad loan}

Similar to Figure 6, we analyze the impact of cash deposit on the rate of the bad loan for the financing platform. The initial capital, mortgage assets, production cost, market risk and demand are randomly generated to simulate the bankruptcy rate for several newsvendors under different circumstances.

Figure 7a shows that cash deposit does not obviously reduce the rate of bad loan for the financing platform. With the cash deposit, the gap of the quantity decision for newsvendors is small. This model does not evade the bankruptcy risk of newsvendors with less initial capital. Thus, cash deposit can, only to a certain extent, 


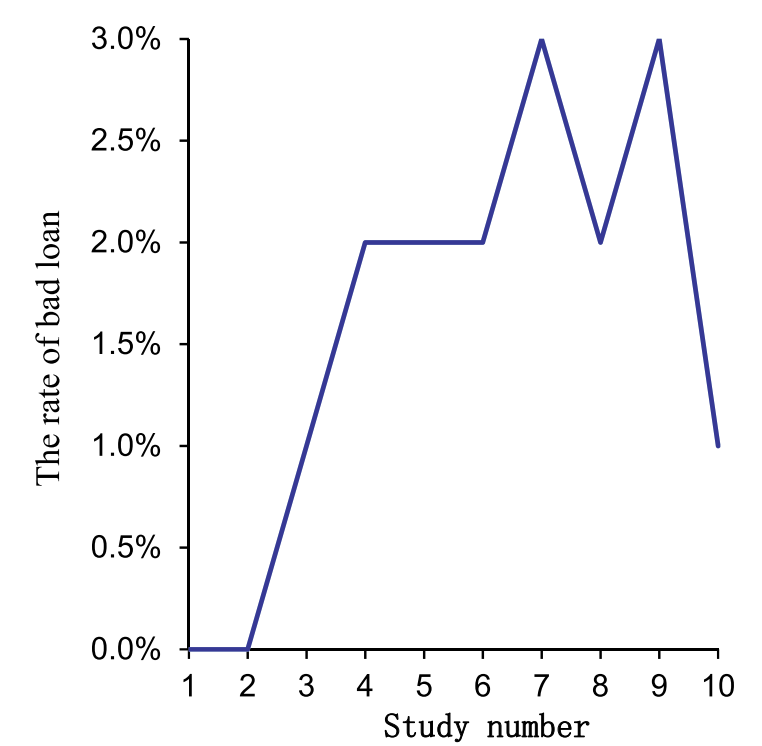

(a)

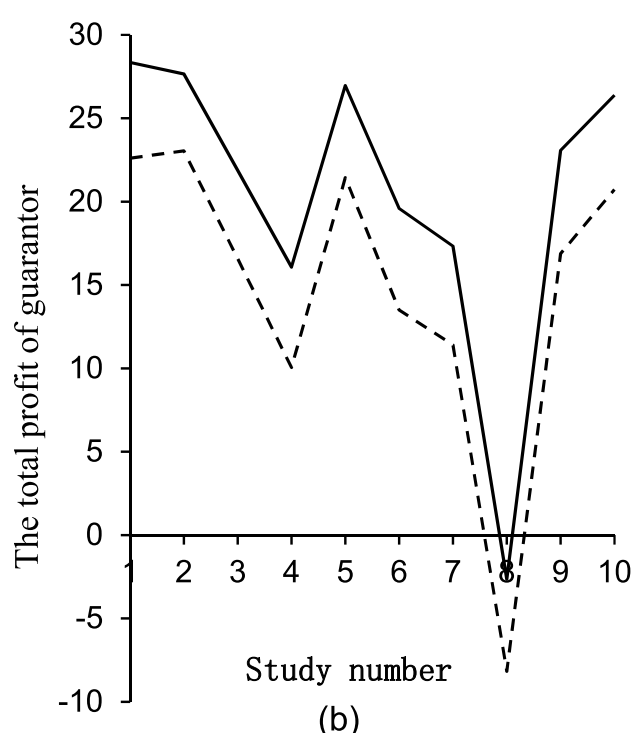

(b)

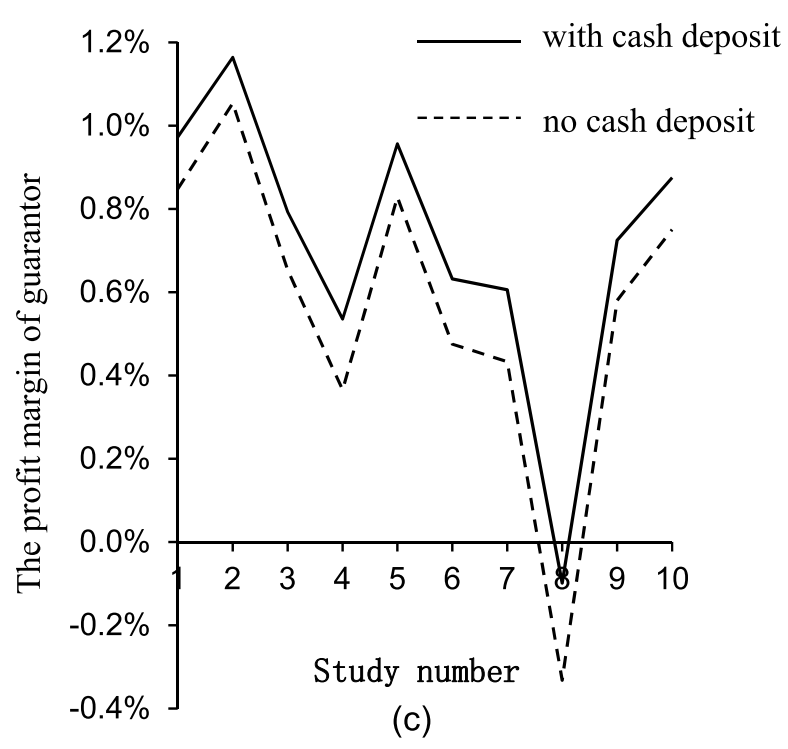

Figure 7 . The impact of financing ceiling on the bad debt rate of financing platform and the total income of guarantor.

reduce the bad loan of the newsvendors, who then face difficulties to gain profit. Therefore, cash deposit is not highly related to the rate of bad loan for the financing platform.

\subsection{Impact of cash deposit on the guarantor's profit}

Figures $7 \mathrm{~b}$ and $7 \mathrm{c}$ show that the cash deposit can increase the guarantor profit and its margins. The profit margin of the guarantor increased but not significantly improved. Thus, the expected guarantor profit is still low, due to the little change from the added cash deposit in the decision about the order quantity. The cash 
deposit can increase the guarantor profit, but cannot effectively ensure its positive value. Figure 7 shows the limitations of cash deposit on risk control mechanism in the supply chain finance.

\section{Conclusions And FUture ReSEARCH}

This study investigates an online financing scheme consisting of a capital-constrained newsvendor, bank, and a guarantor. Two types of risk control mechanism are considered, namely, cash deposit and loan limits set by a maximal profit or a retained profit.

Different from previous literature, this study not only considers the effect of the initial capital of financing enterprises, but also considers that of mortgage assets. The findings are as follows: (1) When the newsvendor has bankruptcy risk, high initial capital and mortgage assets reduce their order quantity; (2) With low newsvendor's initial capital and mortgage assets, the guarantor profit increases with the mortgage asset. This shows that the guarantor should provide guarantees for newsvendors with high mortgage assets to improve their profit level or reduce the risk of repayment.

This study also examines the influence of cash deposit on the newsvendor's quantity decision and the guarantor profit. The findings are as follows: (1) High cash deposit collected leads to less quantity decision of the newsvendor; (2) When the newsvendor has bankruptcy risk and low initial capital, the guarantor profit can increase by collecting cash deposit; (3) When the newsvendor has bankruptcy risk and high initial capital, the guarantor profit decreases by collecting the cash deposit. These findings show that although collecting cash deposit from capital-constrained newsvendors can reduce their excessive quantity decisions, this approach also restricts the optimal quantity decision of newsvendors with high mortgage assets and reduce the guarantor profits. Therefore, the risk control mechanism of cash deposit presents certain limitations.

The value of risk control mechanism of setting the loan limit is also analyzed. The findings are as follows: (1) The loan limit set by a maximum or retained profit increases with the newsvendor's initial capital; (2) With low initial capital, the newsvendor cannot obtain the optimal quantity decision due to the smaller loan limit, which thus becomes the optimal solution. In this case, the guarantor profit increases from the newsvendors initial capital; (3) Setting the loan limit can effectively prevent the guarantor from obtaining negative expected profit and reduce the bankruptcy risk of newsvendors with low initial capital; (4) Comparing the risk control mechanism of the two loan limits, that set by a retained profit can increase the loan amount of newsvendors and increase their financing willingness, but also increase the repayment risk and reduce the expected guarantor profit. Therefore, in practice, the guarantor can choose the appropriate setting of loan limit according to the demands of newsvendors and their own risks and benefits.

Finally, the effect of cash deposit and loan limit on the profits of the financing platform and the guarantor is analyzed through numerical study. The findings are as follows: (1) The cash deposit cannot significantly reduce the rate of bad loan for the bank. The cash deposit can enhance the expected guarantor profit but cannot always ensure a positive value. This finding illustrates that the risk control mechanism of cash deposit in the supply chain finance presents limitations in practice; (2) Setting a loan limit can ensure that the guarantor profit increases and is always positive. When the loan limit is set by a retained profit, the total guarantor profit is slightly less than that of the loan limit that is set by a maximal profit, but the rate of bad loan for the bank is significantly higher than that of the loan limit that is set by a maximal profit, and significantly lower than that without loan limit.

This study is developed based on certain assumptions, which then induces limitations. For example, the risk control policies of cash deposit and loan limit are examined separately. Other hybrid risk control mechanisms based on CVar method can be explored in the future. Pricing strategies in the supply chain are excluded, but considering the operational transactions between newsvendor and suppliers should be worthy of study. Finally, other extensions such as asymmetric information, risk attitude of players, guarantee strategy of insurance companies are recommended for future examination. 


\section{Appendix A. Online supplements}

Proofs for "Risk Control Mechanisms of Third-party Guarantee When Financing Newsvendor":

Proof to Proposition 4.1. (1) When the loan amount is larger than 0 , we must have $B=0$, thus, $(1-\beta) L=$ $c q-B_{0}$. If for any demand $\xi$ satisfying that $B_{T}(\xi)+\alpha A \geq 0$, then the newsvendor has no bankruptcy risk. Consider the worst case, that is, $\xi=0$, we have $B_{T}(0)=\theta\left(B_{0}-c q\right)+v q$. Therefore, it is obvious that $B_{T}(0) \geq 0$ when $q<\hat{q}, B_{T}(0)+\alpha A \geq 0$ when $q \leq \tilde{q}$, and $B_{T}(0)+\alpha A<0$ when $q>\tilde{q}$. All results in the proposition can be obtained.

(2) When demand $\xi<q$, we have $B_{T}(\xi)=(p-v) \xi-(\theta c-v)(q-\hat{q}), \tilde{\Pi}_{f}(q, \xi)=(p-v) \xi-(\theta c-v)(q-\tilde{q})$. Since $p>\theta c$ and $\tilde{q}>\hat{q}$, we have $d_{2}(q)<d_{1}(q)<q$. Thus, when $\hat{q} \leq q \leq \tilde{q}$, if $\xi<d_{1}(q)$, we have $B_{T}(\xi)<0$, that is, the newsvendor will liquidate the mortgage asset to repay the loan, and $\tilde{\Pi}_{f}(q, \xi) \geq 0$ for any $\xi$. $\tilde{\Pi}_{f}(q, \xi)=(p-v) \min \{\xi, q\}-(\theta c-v) q+\theta B_{0}+\alpha A$.

(3) When demand $\xi<q$, we have $\tilde{\Pi}_{f}(q, \xi)=(p-v) \xi-(\theta c-v)(q-\tilde{q})$. Thus, when $q>\tilde{q}$, if $\xi<d_{2}(q)$, we have $B_{T}(\xi)+\alpha A<0$, that is, the newsvendor is bankruptcy. Therefore, when $\xi<d_{2}(q)$, the amount of asset for the newsvendor is 0 . When $\xi \geq d_{2}(q), \tilde{\Pi}_{f}(q, \xi)=(p-v) \min \{\xi, q\}-(\theta c-v) q+\theta B_{0}+\alpha A=$ $(p-v)\left[\min \{\xi, q\}-d_{2}(q)\right]$.

Proof to Proposition 4.2. (1) When the newsvendor has sufficient initial capital, this problem can be seen as a traditional newsvendor problem. Thus, the optimal solution is $Q_{\mathrm{nb}}$.

(2) When $B_{0}<c Q_{\mathrm{nb}}$, the newsvendor can not get the optimal solution of a traditional newsvendor problem. When $q=Q_{\text {bwo }}$, it does not satisfy the condition $B_{0} / c<q \leq \tilde{q}$. Thus, the optimal solution is $q^{*}=B_{0} / c$.

(3) Since $Q_{\text {bwo }} \leq \tilde{q}$, we have $B_{0} \geq \tilde{B}_{0}$. Based on the definition of $\tilde{A}$ and $\tilde{B}_{0}$, it implies that $\tilde{B}_{0}>0$ if $A<\tilde{A}$, otherwise $\tilde{B}_{0} \leq 0$. Therefore, the results of (3) and (4) in Proposition 4.2 can be obtained, respectively.

Proof to Corollary 4.3. (1) By applying the Implicit Function Theorem on equation (4.4) and taking the derivative with respect to $A$ on both sides, we get,

$$
\frac{\mathrm{d} Q_{\mathrm{bwr}}}{\mathrm{d} A}=-\frac{\alpha h\left(d_{2}\left(Q_{\mathrm{bwr}}\right)\right) /(p-v)}{h\left(Q_{\mathrm{bwr}}\right)-\frac{\theta c-v}{p-v} h\left(d_{2}\left(Q_{\mathrm{bwr}}\right)\right)} .
$$

It is obvious that $\mathrm{d} Q_{\mathrm{bwr}} / \mathrm{d} A<0$ since the demand $\xi$ has IFR property.

Similarly, we can have $\mathrm{d} Q_{\mathrm{bwr}} / \mathrm{d} B_{0}<0$.

(2) From the (4) in Proposition 4.2, if $A \geq \tilde{A}$ and $0 \leq B_{0} \leq c Q_{\text {bwo, }}$, the newsvendor's optimal solution is $Q_{\text {bwo }}$ and the newsvendor has no bankruptcy risk in this situation. Similarly, when $B_{0} \geq c Q_{\text {bwo }}$, there is no finance and thus the newsvendor also has no bankruptcy risk.

Proof to Corollary 4.4. (1) It is straightforward to obtain this result and we omit here.

(2) When $B_{0} / c<q \leq \tilde{q}$, the loan amount is $L=\left(L_{0}\right) /(1-\beta)$. However, there is no bankruptcy risk in this situation. Consequently, the profit of the guarantor is $R_{g}\left(c q-B_{0}\right) /(1-\beta)$.

(3) Similarly, we can obtain that the profit of the guarantor is $R_{g}\left(c q-B_{0}\right) /(1-\beta)-(p-v)\left[d_{2}(q)-\xi\right]$.

Proof to Proposition 4.5. First, we focus on the relationship between $Q_{g}$ and $Q_{\text {bwr }}$ when $B_{0}=0$.

When $B_{0}=0$, we have $Q_{g}=\frac{\alpha A}{\theta c-v}+q_{g}, \bar{F}\left(Q_{\text {bwr }}\right)=\frac{\theta c-v}{p-c} \bar{F}\left(\frac{\theta c-v}{p-c}\left(Q_{\text {bwr }}-\frac{\alpha A}{\theta c-v}\right)\right)$. With the definition of $A_{0}^{*}$, we can get: $Q_{g}=Q_{\text {bwr }}$ when $A=A_{0}^{*}$ and $B_{0}=0$.

From Corollary 4.3, when $B_{0}=0$, we have $Q_{\text {bwr }}$ is decreasing in $A$. However, $Q_{g}$ is increasing in $A$. Consequently, when $B_{0}=0$, if $0 \leq A<A_{0}^{*}$, we have $Q_{g}<Q_{\text {bwr }}$; if $A \geq A_{0}^{*}$, we have $Q_{g} \geq Q_{\text {bwr }}$.

According the aforementioned analysis, we can know: 
(1) When $0 \leq A<A_{0}^{*}$, for a given $A$, when $B_{0}=0, Q_{g}<Q_{\mathrm{bwr}}$. When $B_{0}=\tilde{B}_{0}, Q_{g}>Q_{\mathrm{bwr}}$. Since $Q_{g}$ is increasing in $B_{0}$ and $Q_{\mathrm{bwr}}$ is decreasing in $B_{0}$, therefore, there exists a point $B_{0}^{*} \in\left[0, \tilde{B}_{0}\right]$ satisfying $Q_{g}=Q_{\mathrm{bwr}}$.

(2) When $A \geq A_{0}^{*}$, we can obtain: when $B_{0}=0, Q_{g} \geq Q_{\mathrm{bwr}}$; when $B_{0}=\tilde{B}_{0}, Q_{g}>Q_{\mathrm{bwr}}$. Since $Q_{g}$ is increasing in $B_{0}$ and $Q_{\mathrm{bwr}}$ is decreasing in $B_{0}$, therefore, for any $B_{0}, Q_{g} \geq Q_{\mathrm{bwr}}$ always holds.

(3) For a given $A$, when $B_{0} \geq \tilde{B}_{0}$, we have $Q_{\mathrm{bwr}}=Q_{\mathrm{bwo}} \leq \tilde{q}$, since $Q_{g}>\tilde{q}, Q_{g}>Q_{\text {bwo }}$ always holds.

Proof to Corollary 4.6.

$$
\frac{\partial \Pi_{g}\left(Q_{\mathrm{bwr}}\right)}{\partial A}=\alpha F\left(d_{2}\left(Q_{\mathrm{bwr}}\right)\right)+(\theta c-v)\left[\frac{c R_{g}}{(1-\beta)(\theta c-v)}-F\left(d_{2}\left(Q_{\mathrm{bwr}}\right)\right)\right] \frac{\mathrm{d} Q_{\mathrm{bwr}}}{\mathrm{d} A} .
$$

From Proposition 4.5, when $0 \leq A<A_{0}^{*}$ and $B_{0} \in\left[0, B_{0}^{*}\right], Q_{\mathrm{bwr}}>Q_{g}$, we have $d_{2}\left(Q_{\mathrm{bwr}}\right)>d_{2}\left(Q_{g}\right)$, that is, $F\left(d_{2}\left(Q_{\mathrm{bwr}}\right)\right)>\frac{R_{g} c}{(1-\beta)(p-v)}$. Thus, $\partial \Pi_{g}\left(Q_{\mathrm{bwr}}\right) / \partial A>0$.

Proof to Lemma 4.7. From Proposition 4.2, when $A<\tilde{A}$ and $\tilde{B}_{0} \leq B_{0} \leq c Q_{\mathrm{bwo}}$ or $A \geq \tilde{A}$ and $0 \leq B_{0} \leq c Q_{\mathrm{bwo}}$, the newsvendor has no bankruptcy risk and the optimal solution is $Q_{\text {bwo }}$. It is straightforward that $Q_{\text {bwo }}$ is decreasing in $\beta$, thus we have $Q_{\text {bwo }} \leq Q_{\text {bwo }}^{0}$.

When $A<\tilde{A}$ and $0 \leq B_{0}<\tilde{B}_{0}$, the newsvendor has bankruptcy risk and the optimal solution is $Q_{\mathrm{bwr}}$. Since $\theta$ is increasing is $\beta$, to make it easy to solve, we focus on the relationship between $Q_{\mathrm{bwr}}$ and $\theta$. By applying the Implicit Function Theorem on equation (4.4) and taking the derivative with respect to $\theta$ on both sides, we get,

$$
\frac{\mathrm{d} Q_{\mathrm{bwr}}}{\mathrm{d} \theta}=\frac{c\left[1-\left(Q_{\mathrm{bwr}}-\frac{B_{0}}{c}\right) \frac{\theta c-v}{p-v} h\left(d_{2}\left(Q_{\mathrm{bwr}}\right)\right)\right]}{(\theta c-v)\left[\frac{\theta c-v}{p-v} h\left(d_{2}\left(Q_{\mathrm{bwr}}\right)\right)-h\left(Q_{\mathrm{bwr}}\right)\right]} .
$$

Since $h(x)$ is an increasing function and $Q_{\mathrm{bwr}}>d_{2}\left(Q_{\mathrm{bwr}}\right)$, we have the denominator of $\frac{\mathrm{d} Q_{\mathrm{bwr}}}{\mathrm{d} \theta}$ is $(\theta c-$ $v)\left[\frac{\theta c-v}{p-v} h\left(d_{2}\left(Q_{\mathrm{bwr}}\right)\right)-h\left(Q_{\mathrm{bwr}}\right)\right]<0$. Next we analyze that whether the value of the numerator of $\frac{\mathrm{d} Q_{\mathrm{bwr}}}{\mathrm{d} \theta}$ is positive or negative.

We first consider the situation where $B_{0}=0, A=0$. In this case, we have $\tilde{q}=0$ and then $d_{2}\left(Q_{\mathrm{bwr}}\right)=$ $\frac{\theta c-v}{p-v} Q_{\mathrm{bwr}}$. From equation (4.4), we have $Q_{\mathrm{bwr}} \bar{F}\left(Q_{\mathrm{bwr}}\right)=\frac{\theta c-v}{p-v} Q_{\mathrm{bwr}} \bar{F}\left(d_{2}\left(Q_{\mathrm{bwr}}\right)\right)$, that is, $Q_{\mathrm{bwr}} \bar{F}\left(Q_{\mathrm{bwr}}\right)=$ $d_{2}\left(Q_{\mathrm{bwr}}\right) \bar{F}\left(d_{2}\left(Q_{\mathrm{bwr}}\right)\right)$.

We define $V(Q)=Q \bar{F}(Q), H(Q)=Q h(Q)$. Thus, we have $V\left(Q_{\mathrm{bwr}}\right)=V\left(d_{2}\left(Q_{\mathrm{bwr}}\right)\right)$.

Then we study the property about $V(Q) . V(Q)$ is a uni-modular function because $V^{\prime}(Q)=\bar{F}(Q)(1-H(Q))$ and $V^{\prime}(Q)$ is decreasing in $Q$. Thus, when $V^{\prime}(\tilde{Q})=0$, we have $H(\tilde{Q})=1$ and $V(\tilde{Q})$ obtains the maximal value. Moreover, when $0<Q<\tilde{Q}, V(Q)$ is increasing. When $Q \geq \tilde{Q}, V(Q)$ is decreasing. Since $V\left(Q_{\text {bwr }}\right)=$ $V\left(d_{2}\left(Q_{\mathrm{bwr}}\right)\right)$ and $Q_{\mathrm{bwr}} \geq \tilde{Q} \geq d_{2}\left(Q_{\mathrm{bwr}}\right)$, we have $H\left(d_{2}\left(Q_{\mathrm{bwr}}\right)\right) \leq 1 \leq H\left(Q_{\mathrm{bwr}}\right)$. Therefore, when $B_{0}=0$ and $A=0$, we have $1-Q_{\mathrm{bwr}} \frac{\theta c-v}{p-v} h\left(d_{2}\left(Q_{\mathrm{bwr}}\right)\right)=1-H\left(Q_{\mathrm{bwr}}\right)>0$, and $\mathrm{d} Q_{\mathrm{bwr}} / \mathrm{d} \theta<0$.

From Corollary $4.3, Q_{\mathrm{bwr}}$ is decreasing in $B_{0}$ and $A$. Obviously, $\tilde{q}$ is increasing in $B_{0}$ and $A$. Therefore, $d_{2}\left(Q_{\mathrm{bwr}}\right)$ is decreasing in $B_{0}$ and $A$. For any given $A<\tilde{A}$ and $0 \leq B_{0}<\tilde{B}_{0}$, both the value of $Q_{\mathrm{bwr}}-$ $\frac{B_{0}}{c}$ and $h\left(d_{2}\left(Q_{\mathrm{bwr}}\right)\right)$ are lower than that when $B_{0}=0, A=0$. Based on the aforementioned analysis, we have proved that $1-\left(Q_{\mathrm{bwr}}-\frac{B_{0}}{c}\right) \frac{\theta c-v}{p-v} h\left(d_{2}\left(Q_{\mathrm{bwr}}\right)\right)>0$ when $B_{0}=0, A=0$. Consequently, we have $1-$ $\left(Q_{\text {bwr }}-\frac{B_{0}}{c}\right) \frac{\theta c-v}{p-v} h\left(d_{2}\left(Q_{\text {bwr }}\right)\right)>0$ for any $A$ and $B_{0}$ satisfying $A<\tilde{A}$ and $0 \leq B_{0}<\tilde{B}_{0}$. Above all, we have $\mathrm{d} Q_{\mathrm{bwr}} / \mathrm{d} \theta<0$.

Proof to Proposition 4.8. (1) When $A<\tilde{A}$ and $\tilde{B}_{0} \leq B_{0} \leq c Q_{\mathrm{bwo}}$ or $A>\tilde{A}$ and $0 \leq B_{0}<c Q_{\mathrm{bwo}}$, the newsvendor's optimal solution is $Q_{\mathrm{bwo}}$. From Lemma 4.7, $Q_{\mathrm{bwo}}$ is decreasing in $\beta$. It is straightforward that $R_{g}\left(c Q_{\text {bwo }}-B_{0}\right)<R_{g}\left(c Q_{\text {bwo }}^{0}-B_{0}\right)$. 
(2) When $0 \leq A<A_{0}^{*}$ and $B_{0}^{\beta} \leq B_{0}<B_{0}^{*}$, the newsvendor's optimal solution is $Q_{\mathrm{bwr}}$. The profit of the guarantor is $\Pi_{g}\left(Q_{\mathrm{bwr}}\right)=\frac{R_{g}}{1-\beta}\left(c Q_{\mathrm{bwr}}-B_{0}\right)-(p-v) d_{2}\left(Q_{\mathrm{bwr}}\right) F\left(d_{2}\left(Q_{\mathrm{bwr}}\right)\right)+(p-v) \int_{0}^{d_{2}\left(Q_{\mathrm{bwr}}\right)} \xi f(\xi) \mathrm{d} \xi$. Taking the derivative with respect to $\beta$ on $\Pi_{g}\left(Q_{\mathrm{bwr}}\right)$, we have

$$
\frac{\partial \Pi_{g}\left(Q_{\mathrm{bwr}}\right)}{\partial \beta}=\frac{R\left(c Q_{\mathrm{bwr}}-B_{0}\right)}{(1-\beta)^{2}}\left[\frac{R_{g}}{R}-F\left(d_{2}\left(Q_{\mathrm{bwr}}\right)\right)\right]+(\theta c-v) \frac{\partial Q_{\mathrm{bwr}}}{\partial \beta}\left[\frac{c R_{g}}{(1-\beta)(\theta c-v)}-F\left(d_{2}\left(Q_{\mathrm{bwr}}\right)\right)\right] .
$$

In the case where $0 \leq A<A_{0}^{*}$. From Proposition 4.5, there exists a point $B_{0}^{*} \in\left[0, \tilde{B}_{0}\right]$, satisfying: if $0 \leq B_{0} \leq B_{0}^{*}$, then $Q_{g} \leq Q_{\mathrm{bwr}}$ and $d_{2}\left(Q_{g}\right) \leq d_{2}\left(Q_{\mathrm{bwr}}\right)$. Referring to the definition of $Q_{g}$, we can get $\frac{c R_{g}}{(1-\beta)(\theta c-v)} \leq F\left(d_{2}\left(Q_{\text {bwr }}\right)\right)$.

For $c>v$, it is obvious that $\frac{c R_{g}}{(1-\beta)(\theta c-v)}<\frac{R_{g}}{R}$. Since $F\left(d_{2}\left(Q_{\mathrm{bwr}}\right)\right)$ is decreasing in $B_{0}$, thus, we can obtain: when $B_{0}^{\beta} \leq B_{0} \leq B_{0}^{*}, \frac{c R_{g}}{(1-\beta)(\theta c-v)} \leq F\left(d_{2}\left(Q_{\mathrm{bwr}}\right)\right) \leq \frac{R_{g}}{R}$. Moreover, $\frac{c Q_{\mathrm{bwr}}-B_{0}}{(1-\beta)^{2}}>0$ and $\frac{\partial Q_{\mathrm{bwr}}}{\partial \beta} \leq 0$, consequently, we have $\frac{\partial \Pi_{g}\left(Q_{\mathrm{bwr}}\right)}{\partial \beta}>0$.

Proof to Corollary 5.1. Based on the aforementioned analysis, we have $Q_{g}=\frac{\theta B_{0}+\alpha A}{\theta c-v}+q_{g}$. Given $p, c, R, v$ and $\beta, q_{g}$ is constant. Thus, it is straightforward that $Q_{g}$ is increasing in $B_{0}$.

Proof to Corollary 5.2. Since

$$
R_{g}\left(c Q_{g}^{u}-B_{0}\right)-(p-v) d_{2}\left(Q_{g}^{u}\right) F\left(d_{2}\left(Q_{g}^{u}\right)\right)+(p-v) \int_{0}^{d_{2}\left(Q_{g}^{u}\right)} \xi f(\xi) \mathrm{d} \xi=R_{f}\left(c Q_{g}^{u}-B_{0}\right),
$$

applying the Implicit Function Theorem on this equation and taking the derivative with respect to $B_{0}$ on both sides, we get,

$$
\frac{\mathrm{d} Q_{g}^{u}}{\mathrm{~d} B_{0}}=\frac{-\theta F\left(d_{2}\left(Q_{g}^{u}\right)\right)+\left(R_{g}-R_{f}\right)}{c\left(R_{g}-R_{f}\right)-[\theta c-v] F\left(d_{2}\left(Q_{g}^{u}\right)\right)} .
$$

Because $Q_{g}^{u}>Q_{g}$, it is easy to obtain that $F\left(d_{2}\left(Q_{g}^{u}\right)\right)>F\left(d_{2}\left(Q_{g}\right)\right)$. Substituting it into above calculation, we can obtain $\frac{d Q_{g}^{u}}{d B_{0}}>0$. Thus, $Q_{g}^{u}$ is increasing in $B_{0}$.

Proof to Proposition 5.3. (1) When the upper bound of limit amount is $Q_{g}$ and $B_{0}<B_{0}^{*}$, the newsvendor's quantity decision is $Q_{g}$, thus we have

$$
\partial \Pi_{g}\left(B_{0}\right) / \partial B_{0}=\left[c R_{g}-(\theta c-v) F\left(d_{2}\left(Q_{g}\right)\right)\right] \frac{\mathrm{d} Q_{g}}{\mathrm{~d} B_{0}}+\left[\theta F\left(d_{2}\left(Q_{g}\right)\right)-R_{g}\right] .
$$

Since $F\left(d_{2}\left(Q_{g}\right)\right)=\frac{c R_{g}}{\theta c-v}$, then we have $\theta F\left(d_{2}\left(Q_{g}\right)\right)-R_{g}=\theta \frac{c R_{g}}{\theta c-v}-R_{g}=\frac{v R_{g}}{\theta c-v}>0$. Consequently, $\partial \Pi_{g}\left(B_{0}\right) / \partial B_{0}>0$.

(2) Similar with (1), in this situation, the newsvendor's quantity decision is $Q_{g}^{u}$,

$$
\frac{\partial \Pi_{f}\left(B_{0}\right)}{\partial B_{0}}=\left[c R_{g}-(\theta c-v) F\left(d_{2}\left(Q_{g}^{u}\right)\right)\right] \mathrm{d} Q_{g}^{u} / \mathrm{d} B_{0}+\left[\theta F\left(d_{2}\left(Q_{g}^{u}\right)\right)-R_{g}\right] .
$$

Substituting the equation (A.1) into the equation (A.2), we have $\frac{\partial \Pi_{f}\left(B_{0}\right)}{\partial B_{0}}=-\frac{v F\left(d_{2}\left(Q_{g}^{u}\right)\right)}{c\left(R_{g}-R_{f}\right)-(\theta c-v) F\left(d_{2}\left(Q_{g}^{u}\right)\right)} R_{f}$. Because $Q_{g}^{u}>Q_{g}$, it is easy to obtain that $d_{2}\left(Q_{g}^{u}\right)>d_{2}\left(Q_{g}\right), F\left(d_{2}\left(Q_{g}^{u}\right)\right)>F\left(d_{2}\left(Q_{g}\right)\right)=\frac{c R_{g}}{\theta c-v}$. Thus, $\frac{\partial \Pi_{f}\left(B_{0}\right)}{\partial B_{0}}>0$. 
Proof to Proposition 5.4. (1) When the loan limit is $Q_{g}$, when $0<B_{0}<B_{0}^{*}$, the probability of the newsvendor being bankruptcy is $P_{r}\left\{\xi \leq d_{2}\left(Q_{g}\right)\right\}=F\left(d_{2}\left(Q_{g}\right)\right)$. Since $F\left(d_{2}\left(Q_{g}\right)\right)=\frac{c R_{g}}{\theta c-v}$, it is obvious that $P_{r}\{\xi \leq$ $\left.d_{2}\left(Q_{g}\right)\right\}$ is independent in $B_{0}$.

When $B_{0}^{*}<B_{0}<\tilde{B}_{0}$, the newsvendor's quantity decision is $Q_{\mathrm{bwr}}$. Thus, $P_{r}\left\{\xi \leq d_{2}\left(Q_{\mathrm{bwr}}\right)\right\}=F\left(d_{2}\left(Q_{\mathrm{bwr}}\right)\right)$, from the equation (4.4), we have $F\left(d_{2}\left(Q_{\mathrm{bwr}}\right)\right)=\frac{p-v}{\theta c-v} F\left(Q_{\mathrm{bwr}}\right)+\frac{\theta c-p}{\theta c-v}$. From Corollary $4.3, d Q_{\mathrm{bwr}} / d B_{0}<0$, thus $F\left(d_{2}\left(Q_{\mathrm{bwr}}\right)\right)$ is decreasing in $B_{0}$.

(2) Since $Q_{g}<Q_{g}^{u}$, we have $d_{2}\left(Q_{g}\right)<d_{2}\left(Q_{g}^{u}\right)$. It is straightforward that $F\left(d_{2}\left(Q_{g}\right)\right)<F\left(d_{2}\left(Q_{g}^{u}\right)\right)$.

Acknowledgements. This research is supported by the Natural Science Foundation of China (Nos. 71672179, 71872051), Foundation of Philosophy and Social Science Research in Colleges and Universities in Jiangsu Province (No. 2018SJA0325), 2019 Qinglan Project of Jiangsu Province in China, Foundation of Audit Information Engineering and Technology Collaborative Innovation Center in Nanjing Audit University (No. 099810401) and Priority Academic Program Development of Jiangsu Higher Eduction Institutions.

\section{REFERENCES}

[1] D. Akkaya, K. Bimpikis and H. Lee, Government interventions to promote agricultural innovation. Manuf. Serv. Oper. Manage. 23 (2021) 437-452.

[2] S. Alizamir, F. Iravani and H. Mamani, An analysis of price vs. revenue protection: government subsidies in the agriculture industry. Manage. Sci. 65 (2019) 32-49.

[3] S. An, B. Li, D.P. Song and X. Chen, Green credit financing versus trade credit financing in a supply chain with carbon emission limits. Eur. J. Oper. Res. 292 (2021) 125-142.

[4] M.A. Barach, J.M. Golden and J.J. Horton, Steering in online markets: the role of platform incentives and credibility. Manage. Sci. 66 (2020) 4047-4070.

[5] J.A. Buzacott and R.Q. Zhang, Inventory management with asset-based financing. Manage. Sci. 50 (2004) $1274-1292$.

[6] X. Chen, G.G. Cai and J.-S. Song, The cash flow advantages of 3pls as supply chain orchestrators. Manuf. Serv. Oper. Manage. 21 (2016) 435-451.

[7] X. Chen, Q. Lu and G.G. Cai, Buyer financing in pull supply chains: Zero-interest early payment or in-house factoring? Prod. Oper. Manage. 29 (2020) 2307-2325.

[8] J. Chod, Inventory, risk shifting, and trade credit. Manage. Sci. 63 (2017) 3207-3225.

[9] S. Deng, C. Gu, G.G. Cai and Y. Li, Financing multiple heterogeneous suppliers in assembly systems: buyer finance vs. bank finance. Manuf. Serv. Oper. Manage. 20 (2018) 53-69.

[10] FDIC, Federal Deposit Insurance Corporation Database. Retrieved from www.fdic.gov (2018).

[11] B. Huang, A. Wu and D. Chiang, Supporting small suppliers through buyer-backed purchase order financing. Int. J. Prod. Res. 56 (2018) 1-24.

[12] W. Jin, Q. Zhang and J. Luo, Non-collaborative and collaborative financing in a bilateral supply chain with capital constraints. Omega 88 (2019) 210-222.

[13] A. Khanna, P. Gautam, B. Sarkar and C.K. Jaggi, Integrated vendor-buyer strategies for imperfect production systems with maintenance and warranty policy. RAIRO: OR 54 (2020) 435-450.

[14] P. Kouvelis and W. Zhao, The newsvendor problem and price-only contract when bankruptcy costs exist. Prod. Oper. Manage. 20 (2011) 921-936.

[15] P. Kouvelis and W. Zhao, Financing the newsvendor: supplier vs. bank, and the structure of optimal trade credit contracts. Oper. Res. 60 (2012) 566-580.

[16] B. Li, S. An and D. Song, Selection of financing strategies with a risk-averse supplier in a capital-constrained supply chain. Transp. Res. Part E: Logistics Transp. Rev. 118 (2018) 163-183.

[17] Q. Lu, J. Gu and J. Huang, Supply chain finance with partial credit guarantee provided by a third-party or a supplier. Comput. Ind. Eng. 135 (2019) 440-455.

[18] P. Luo, H. Wang and Z. Yang, Investment and financing for smes with a partial guarantee and jump risk. Eur. J. Oper. Res. 249 (2016) 1161-1168.

[19] Y. Luo, Q. Wei, Q. Ling and B. Huo, Optimal decision in a green supply chain: Bank financing or supplier financing. J. Cleaner Prod. 271 (2020) 122090.

[20] H. Peura, S.A. Yang and G. Lai, Trade credit in competition: a horizontal benefit. Manuf. Serv. Oper. Manage. 19 (2017) 263-289.

[21] X. Qian and T.L. Olsen, Operational and financial decisions within proportional investment cooperatives. Manuf. Serv. Oper. Manage. 22 (2020) 545-561.

[22] B. Sarkar and S. Saren, Product inspection policy for an imperfect production system with inspection errors and warranty cost. Eur. J. Oper. Res. 248 (2016) 263-271. 
[23] Shi, China MSME Finance Report (2017). Access from www.smefinance.forum.org/post/china-msme-finance-report-2016. (Accessed 10 March 2019) (2017).

[24] C.S. Tang, Y. Wang and Z. Ming, The implications of utilizing market information and adopting agricultural advice for farmers in developing economies. Prod. Oper. Manage. 24 (2015) 1197-1215.

[25] C.S. Tang, M.S. Sodhi and M. Formentini, An analysis of partially-guaranteed-price contracts between farmers and agri-food companies. Eur. J. Oper. Res. 254 (2016) 1063-1073.

[26] T.I. Tunca and W. Zhu, Buyer intermediation in supplier finance. Manage. Sci. 64 (2018) 5631-5650.

[27] US Small Business Administration, United States's 2018 small business profile. Accessed from www.sba.gov/sites/default/ files/advocacy/2018-Small-Business-Profiles-US.pdf. (Accessed 10 March 2019) (2018).

[28] N. Yan, B. Sun, H. Zhang and C. Liu, A partial credit guarantee contract in a capital-constrained supply chain: financing equilibrium and coordinating strategy. Int. J. Prod. Econ. 173 (2016) 122-133.

[29] B. Yan, G. Liu, Z. Zhang and C. Yan, Optimal financing and operation strategy of fresh agricultural supply chain. Aust. J. Agric. Res. Econ. 64 (2020) 776-794.

[30] J. Zhan, S. Li and X. Chen, The impact of financing mechanism on supply chain sustainability and efficiency. J. Cleaner Prod. 205 (2018) 407-418.

[31] Z.C. Zhang, H.Y. Xu and K.B Chen, Operational decisions and financing strategies in a capital-constrained closed-loop supply chain. Int. J. Prod. Res. 59 (2021) 4690-4710.

[32] W. Zhou, T. Lin and G.G. Cai, Guarantor financing in a four-party supply chain game with leadership influence. Prod. Oper. Manage. 29 (2020) 2035-2056.

[33] Y.W. Zhou, Z.L. Wen and X. Wu, A single-period inventory and payment model with partial trade credit. Comput. Ind. Eng. 90 (2015) 132-145. 